Delft University of Technology

\title{
A Pitch-Matched Front-End ASIC With Integrated Subarray Beamforming ADC for Miniature 3-D Ultrasound Probes
}

Chen, Chao; Chen, Zhao; Bera, Deep; Noothout, Emile; Chang, Zu Yao; Tan, Mingliang; Vos, Hendrik J.; Bosch, Johan G.; Verweij, Martin D.; de Jong, Nico

DOI

10.1109/JSSC.2018.2864295

Publication date

2018

Document Version

Accepted author manuscript

Published in

IEEE Journal of Solid-State Circuits

\section{Citation (APA)}

Chen, C., Chen, Z., Bera, D., Noothout, E., Chang, Z. Y., Tan, M., Vos, H. J., Bosch, J. G., Verweij, M. D., de Jong, N., \& Pertijs, M. A. P. (2018). A Pitch-Matched Front-End ASIC With Integrated Subarray

Beamforming ADC for Miniature 3-D Ultrasound Probes. IEEE Journal of Solid-State Circuits, 53(11), 30503064. https://doi.org/10.1109/JSSC.2018.2864295

Important note

To cite this publication, please use the final published version (if applicable).

Please check the document version above. 


\title{
A Pitch-Matched Front-End ASIC With Integrated Subarray Beamforming ADC for Miniature 3-D Ultrasound Probes
}

\author{
Chao Chen ${ }^{\circledR}$, Member, IEEE, Zhao Chen ${ }^{\circledR}$, Student Member, IEEE, Deep Bera, Emile Noothout, Zu-Yao Chang, \\ Mingliang Tan ${ }^{\circledR}$, Hendrik J. Vos ${ }^{\circledR}$, Member, IEEE, Johan G. Bosch, Member, IEEE, \\ Martin D. Verweij, Member, IEEE, Nico de Jong ${ }^{\circledR}$, Member, IEEE, \\ and Michiel A. P. Pertijs, Senior Member, IEEE
}

\begin{abstract}
This paper presents a front-end applicationspecified integrated circuit (ASIC) integrated with a 2-D PZT matrix transducer that enables in-probe digitization with acceptable power dissipation for the next-generation endoscopic and catheter-based 3-D ultrasound imaging systems. To achieve power-efficient massively parallel analog-to-digital conversion (ADC) in a 2-D array, a 10-bit $30 \mathrm{MS} / \mathrm{s}$ beamforming ADC that merges the subarray beamforming and digitization functions in the charge domain is proposed. It eliminates the need for costly intermediate buffers, thus significantly reducing both power consumption and silicon area. Self-calibrated charge references are implemented in each subarray to further optimize the system-level power efficiency. High-speed datalinks are employed in combination with the subarray beamforming scheme to realize a 36-fold channel-count reduction and an aggregate output data rate of $6 \mathrm{~Gb} / \mathrm{s}$ for a prototype receive array of $24 \times 6$ elements. The ASIC achieves a record power efficiency of $0.91 \mathrm{~mW} / \mathrm{element}$ during receive. Its functionality has been demonstrated in both electrical and acoustic imaging experiments.
\end{abstract}

Index Terms-3-D ultrasound imaging, charge-sharing successive approximation register (SAR) analog-to-digital conversion (ADC), in-probe digitization, miniature probes, subarray beamforming, ultrasound front-end application-specified integrated circuit (ASIC).

Manuscript received March 12, 2018; revised July 6, 2018; accepted July 26, 2018. This work was supported in part by the Dutch Technology Foundation STW, which is part of the Netherlands Organization for Scientific Research, and in part by the Ministry of Economic Affairs. This paper was approved by Guest Editor Chung-Yu Wu. (Chao Chen and Zhao Chen contributed equally to this work.) (Corresponding author: Chao Chen.)

C. Chen was with the Electronic Instrumentation Laboratory, Delft University of Technology, 2628 CD Delft, The Netherlands. He is now with Butterfly Network Inc., Guilford, CT 06437 USA (e-mail: chaochen.sscs@gmail.com).

Z. Chen, Z.-Y. Chang, M. Tan, and M. A. P. Pertijs are with the Electronic Instrumentation Laboratory, Delft University of Technology, 2628 CD Delft, The Netherlands.

D. Bera was with the Department of Biomedical Engineering, Thoraxcenter, Erasmus Medical Center, 3015 CN Rotterdam, The Netherlands. He is now with Philips India Limited, Bengaluru 560045, India.

E. Noothout is with the Laboratoy of Acoustical Wavefield Imaging, Delft University of Technology, 2628 CJ Delft, The Netherlands.

H. J. Vos, M. D. Verweij, and N. de Jong are with the Laboratory of Acoustical Wavefield Imaging, Delft University of Technology, 2628 CJ Delft, The Netherlands, and also with the Department of Biomedical Engineering, Thoraxcenter, Erasmus Medical Center, 3015 CN Rotterdam, The Netherlands.

J. G. Bosch is with the Department of Biomedical Engineering, Thoraxcenter, Erasmus Medical Center, 3015 CN Rotterdam, The Netherlands.

Color versions of one or more of the figures in this paper are available online at http://ieeexplore.ieee.org.

Digital Object Identifier 10.1109/JSSC.2018.2864295

\section{INTRODUCTION}

D ATA acquisition from 2-D transducer arrays has become one of the main challenges for the development of endoscopic and catheter-based 3-D ultrasound imaging devices, such as transesophageal echocardiography (TEE) [1], intracardiac echocardiography (ICE) [2], and intravascular ultrasound (IVUS) [3], [4] probes. The main obstacle lies in the mismatch between the large number of transducer elements needed for 3-D imaging and the limited number of cables that can be accommodated in these systems. Recent advances in transducer-on-CMOS integration methods [5], [6] have enabled the use of front-end application-specified integrated circuits (ASICs) performing signal conditioning and data reduction close to the transducer. The concept of subarray beamforming [7], which divides the transducer array in subarrays and combines the signals received by the elements in each subarray by a local delay-and-sum operation, is capable of reducing the channel count by an order of magnitude. This has been successfully demonstrated in several ASIC prototypes [1], [8] and has made it possible to develop probes with $3000+$ transducer elements [9]. However, it is still an arduous engineering problem to assemble hundreds of cables within endoscopes or catheters of $\leq 5 \mathrm{~mm}$ diameter [2]. Such constraints have forced system designers to tradeoff imaging quality against physical dimension and fabrication cost [2].

A variety of efforts have been made in recent years to further reduce the cable count by making better use of the cable capacity. Time-multiplexing in the analog domain [10] allows the signals received by multiple elements to share a single cable. However, the limited bandwidth and transmission-line effects of the micro-coaxial cables result in significant channelto-channel crosstalk [11], [12]. Other analog modulation methods, such as frequency-division multiplexing (FDM) [13], also suffer from the cable non-idealities.

A more radical solution is to move analog-to-digital conversions (ADCs) into the probe and perform the channel reduction in the digital domain, where complex modulated signals can be transmitted with much better robustness against noise, interference, and crosstalk. Moreover, in-probe digitization would open up the possibility to migrate more signal processing 


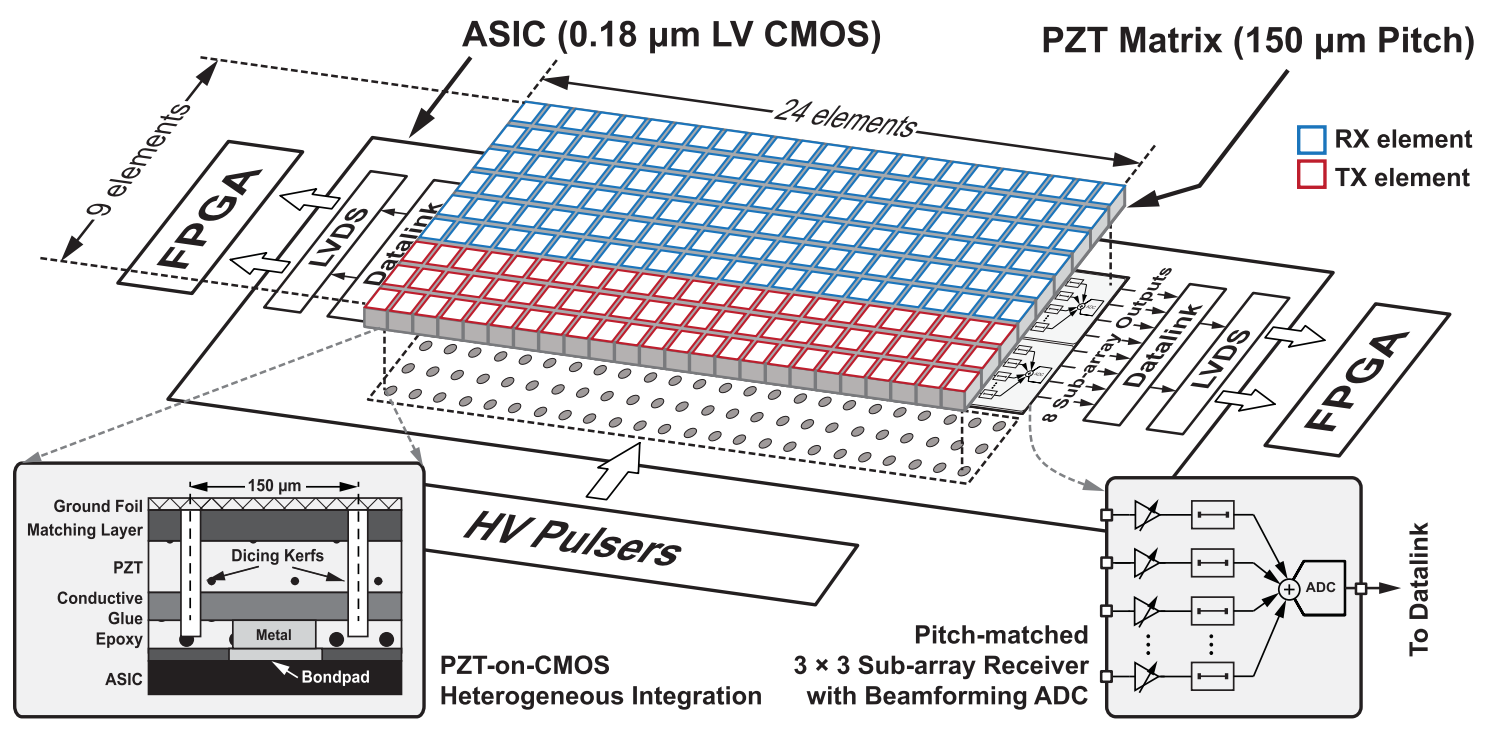

Fig. 1. System overview.

functionalities into the probe, such as post-beamforming [14] and compressive beamforming [15], which are expected to further reduce the channel count. A prerequisite to achieve this goal is an efficient way to implement a massively parallel ADC array within the stringent power and area constraint of miniature ultrasound probes.

Based on the framework of subarray beamforming, in-probe digitization can be realized by digitizing the output of analog subarray beamformers with per-subarray ADCs [16]. Alternatively, digital beamforming with per-element ADCs can be considered [17]-[19]. The latter approach requires $D^{2}$ ADCs and input buffers for a $D \times D$ subarray, plus the associated digital FIFOs for the realization of delays. This is difficult to be directly integrated underneath a pitch-constrained 2-D transducer with an affordable power consumption. In [17], the area problem was addressed by using nanoscale CMOS technologies. Nevertheless, the reported element-level $\Delta \Sigma$ $\mathrm{ADC}$ is larger than the ideal half-wavelength pitch of $150 \mu \mathrm{m}$ at $5 \mathrm{MHz}$, and the power dissipation is more than two orders of magnitude higher than its analog counterpart [1]. Moving the ADC to the output of an analog beamformer helps in reducing the area and power cost for ADC. The work of [16] employs subarray beamformers based on $\mathrm{S} / \mathrm{H}$ circuits followed by a stand-alone Nyquist-rate ADC. The reported silicon area and power are both dominated by the analog beamformer, resulting in a per-channel footprint that is $12 \times$ larger than the dimension of a $5-\mathrm{MHz}$ transducer element and a power consumption far exceeding the heat dissipation constraint for miniature probes [20]. More recently, [21] proposes to embed charge-redistribution successive approximation register (SAR) ADCs with a subarray beamformer, so as to save power. This approach, however, requires $N$ SAR ADCs to implement an $N$-channel beamformer, resulting in a poor area efficiency.

In this paper, we propose an element-pitch-matched ASIC architecture to demonstrate the feasibility of efficient in-probe digitization in miniature ultrasound probes [22]. It provides subarray beamforming for a directly integrated $5-\mathrm{MHz}$ PZT matrix with a half-wavelength element pitch of $150 \mu \mathrm{m}$. In each $3 \times 3$ subarray, a compact Nyquist-rate beamforming $\mathrm{ADC}$ is implemented following the analog front-end (AFE) circuits. By merging the beamforming and digitization functions coherently in the charge domain, there is no need for intermediate ADC buffers, resulting in significant power and area reduction. The outputs of four beamforming ADCs are fed into a high-speed digital serializer on the periphery of the ASIC to reduce the total output channel count by a factor of 4. Thus, the ASIC achieves a 36-fold channelcount reduction, while consuming less than $1 \mathrm{~mW} /$ element in receive mode. The effectiveness of the proposed architecture has been successfully demonstrated in both electrical tests and a 3-D imaging experiment.

This paper is organized as follows. The architecture of the proposed ASIC is discussed in Section II. Section III describes the circuit implementation details of the subarray receiver and the datalink. Section IV presents the experimental setup and results. The conclusion is given in Section V.

\section{System ARChitecture}

\section{A. Overview}

Fig. 1 shows an overview of the proposed system. It consists of a front-end ASIC, a 5-MHz 150- $\mu$ m-pitch PZT matrix, and the associated external electronics. The PZT matrix is constructed from a bulk piezoelectric material (CTS 3202 HD) that is stacked on the ASIC using the PZT-on-CMOS heterogeneous integration process described in [6]. A metallic interconnection layer and a conductive glue layer connect the bottom electrodes of the PZT elements to a bondpad array on the ASIC.

As a proof-of-concept, we use a PZT matrix with a relatively small aperture in this work, while the proposed circuit architecture and layout are both designed to facilitate the future extension to a larger array size, such as the $32 \times 32$ array presented in [1]. The matrix is divided into $3 \times 24$ trans- 


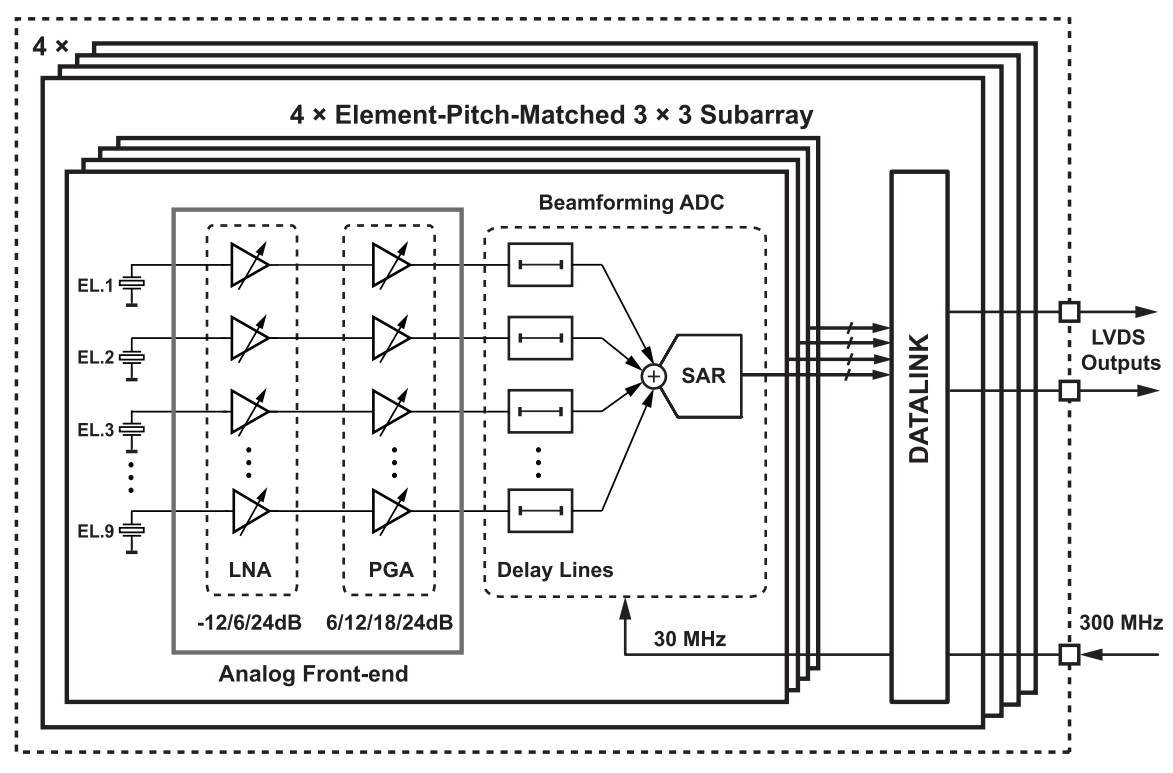

(a) $\mathrm{RX}$ architecture

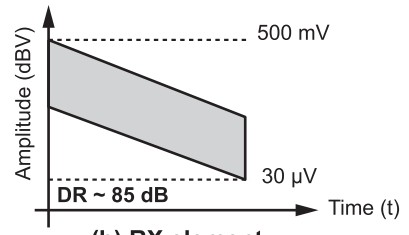

(b) RX element

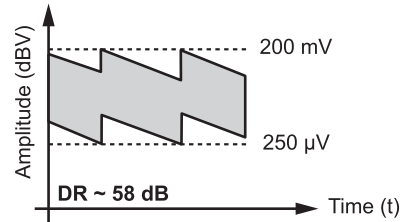

(c) LNA output

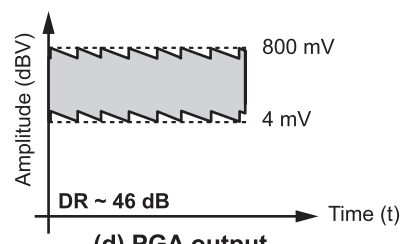

(d) PGA output

Fig. 2. (a) Architecture of receive electronics. (b) Peak-to-peak signal DR at the input of the LNA. (c) Peak-to-peak signal DR at the output of the LNA. (d) Peak-to-peak signal DR at the output of the PGA.

mit $(\mathrm{TX})$ elements and $6 \times 24$ receive $(\mathrm{RX})$ elements. Similar to [1] and [6], the TX elements are directly wired-out to external high-voltage pulsers using metal traces in the ASIC, thus enabling the use of a low-voltage CMOS process [3]. Nevertheless, the concepts presented in this paper are equally applicable to an ASIC in which local TX pulsers are realized using a high-voltage CMOS technology. In this prototype, every three TX elements in the same column are connected together in the ASIC to reduce the overall I/O count, turning them into a 1-D phase array with a broad beam profile in the elevation direction. In a future extension to a larger array, an alternative more symmetrical transmit layout would be preferred, such as the central transmit subarray described in [1]. In RX, subarray beamforming is applied on $3 \times 3$ elements to realize a 9-fold channel reduction, yielding in total 16 subarrays.

Fig. 2(a) shows the architecture of RX electronics in the ASIC. Each subarray receiver consists of a nine-channel AFE that acquires echo signals from the transducer elements and conveys them to programmable delay lines. The output of the nine delay lines are added and digitized in the charge domain by an SAR ADC. The digitized data are transferred to a datalink at the periphery of the ASIC, where the data from four subarrays are combined on a shared low-voltage differential signaling (LVDS) output channel, thus realizing an extra 4-fold channel-count reduction. At the system side, a field-programmable gate array (FPGA) [23] receives and stores the data, which is then transmitted to a PC for image reconstruction.

\section{B. AFE}

The AFE in each channel consists of a low-noise amplifier (LNA) and a programmable-gain amplifier (PGA). Fig. 2(b) illustrates the expected peak-to-peak voltage signal received by a transducer element as a function of time. Note that the time axis, assuming a constant speed of sound, is equivalent to the axial depth. Echoes resulting from deeper scatterers will arrive later, and will be more attenuated, leading to an overall peak-to-peak amplitude range from $30 \mu \mathrm{V}$ to $500 \mathrm{mV}$, while the instantaneous dynamic range (DR), i.e., the ratio between the largest and smallest echoes at a given imaging depth, is about $40 \mathrm{~dB}$. The depth-dependent (and hence time-dependent) attenuation can be compensated by applying time-varying gains in the AFE. Such time-gain compensation (TGC) function is implemented by distributing discrete gain steps between the two stages in the AFE. The LNA provides a high gain for small echo signals from deep scatterers, where the acoustic and electrical noise determine the detection limit, and a lower gain for large echoes from nearby scatterers, where linearity matters. With a gain step of $18 \mathrm{~dB}$, the DR is thus compressed to approximately $58 \mathrm{~dB}$ [Fig. 2(c)]. This is further reduced by the PGA, which provides gain steps of $6 \mathrm{~dB}$, resulting in an output DR of $46 \mathrm{~dB}$, with peak-to-peak amplitudes ranging from 4 to $800 \mathrm{mV}$ [Fig. 2(d)]. This arrangement effectively reduces the noise and DR requirements of the succeeding circuits, while keeping the complexity of the TGC implementation modest, thus bringing significant power and area advantages for the whole system. It can be combined with a fine-gain compensation in the digital back-end to avoid imaging artifacts associated with the gain steps.

\section{Beamforming ADC}

The beamformer in each subarray is similar to [1]. Analog delay lines based on pipeline-operated switched-capacitor memory cells are employed because of their simplicity and flexibility in delay control, as well as their good immunity to process/voltage/temperature (PVT) variations [16], [24]. 
Each delay line consists of eight memory cells operating in a time-interleaved fashion at a sampling rate of $30 \mathrm{MHz}$, corresponding to a delay resolution of $33 \mathrm{~ns}$ and a maximum range of $\sim 233 \mathrm{~ns}$. This delay range allows for pre-steering up to $\pm 37^{\circ}$ in both the azimuthal and elevation directions [6]. The chosen delay quantization causes negligible degradation to the image quality with the aid of the imaging scheme proposed in [25], while requiring only a modest number of memory elements that fit within the available die area. The delay control logic is implemented based on a delay stage index rotator as described in [1], which can be programmed via a built-in serial-peripheral-interface (SPI) embedded in each subarray.

Dynamic focusing in the subarray beamformer is not implemented in this design as it is not required given the relatively small subarray size $(3 \times 3)$ [7], [26]. However, if desired, it can be readily added to the proposed beamforming ADC architecture by adopting the delay extension/skipping approach as described in [9].

As discussed in Section II-A, the signal DR at the output of each AFE channel is $46 \mathrm{~dB}$. Considering the extra $\sqrt{ } 9(9.5 \mathrm{~dB})$ SNR gain provided by a nine-channel beamformer, a 10-bit $\mathrm{ADC}$ is required to achieve an adequate quantization resolution. For an ultrasound transducer with a $\geq 50 \%$ fractional bandwidth, the ADC sampling rate must be 4-10 times the transducer central frequency to maintain a satisfactory sidelobe level [27], which corresponds to 20 to $50 \mathrm{MS} / \mathrm{s}$ for our $5-\mathrm{MHz}$ array. Given these specifications, an SAR ADC stands out as the architecture choice for its superior power efficiency [28].

Most SAR ADCs perform the quantization in the voltage domain [29], [30], while switched-capacitor-based (S/H) delay lines essentially operate in the charge domain. Therefore, a charge-to-voltage conversion is required for driving the ADC. An active summing amplifier was used in [16] to sum the charges stored on the delay-line capacitors $C_{M}$, while an extra voltage buffer drives the ADC [Fig. 3(a)]. To implement a unity voltage gain, the feedback capacitance must be equal to the total memory capacitance involved in each cycle, i.e., $N \times C_{M}$, leading to a considerable power consumption in the amplifier, more than $10 \times$ power of the $\mathrm{ADC}$ in [16]. The summing amplifier can be eliminated by adopting passive charge summation [1] [Fig. 3(b)]. However, the power consumed by the ADC driver is still problematic; it is usually comparable to that of the ADC itself, as a result of the relatively large capacitance it needs to drive during a constrained input sampling time.

To eliminate the ADC driver, we propose to perform the digitization in the charge domain, rather than in the voltage domain [Fig. 3(c)]. This is achieved by sequentially neutralizing the passively summed signal charge with binary-scaled charge references through a successive approximation process. In practice, the charge references can be realized as a precharged capacitor DAC (CDAC) array. By doing so, the beamformer and the digitizer are essentially merged together: the delay lines perform as a multichannel time-interleaved input sampler in a charge-sharing SAR ADC [32]. We will refer to this circuit as a beamforming ADC.

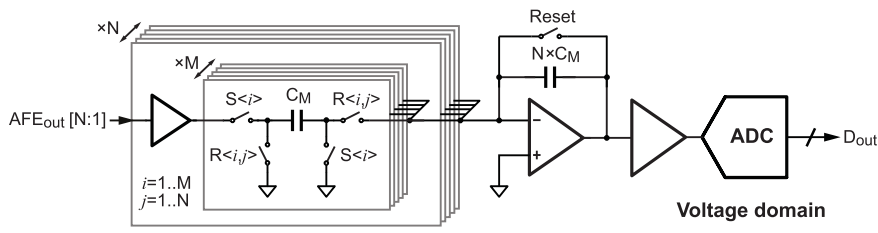

(a)

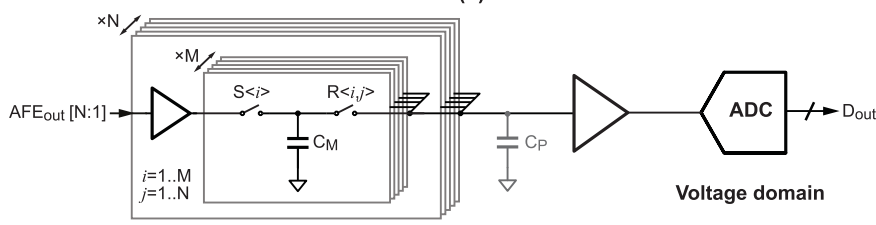

(b)

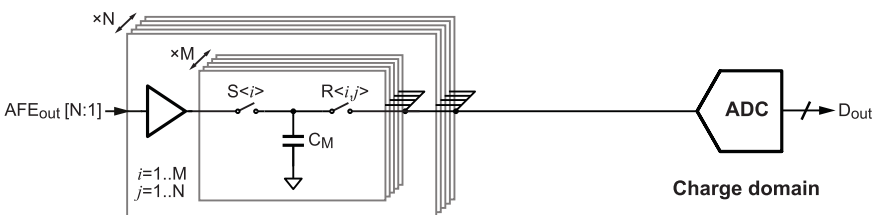

(c)

Fig. 3. Hybrid beamforming with post-analog-beamforming digitizers. (a) $\mathrm{S} / \mathrm{H}$ delay lines with an active summing amplifier. (b) $\mathrm{S} / \mathrm{H}$ delay lines with passive charge summation and active voltage buffer as the ADC driver. (c) Proposed architecture with a charge-domain ADC.

Fig. 4 shows a block diagram of the ADC and its timing diagram. Both the beamforming and the digitization are performed differentially to mitigate the impact of common-mode noise and interference. In each channel, the PGA converts the single-ended LNA output to a differential voltage, which is cyclically sampled and held on memory cells under the control of non-overlapping sampling clocks, $S\langle 1: 8\rangle$. The charge sampled on the memory cells is then released to the summing nodes, $V_{X P}$ and $V_{X N}$, at the rising edges of channel-specific readout clocks, $\mathrm{R}_{k}\langle 1: 8\rangle$, where $k$ ranges from 1 to 9 . The delay of a channel is thus defined by the time interval between the falling edges of its sampling and readout clocks. Before the start of each readout phase, a CDAC array is precharged to a reference voltage $\left(V_{R E F}\right)$. In each readout phase, the successive approximation charge-balancing process starts after a short-time interval reserved for the passive charge redistribution on the joint memory cells. In every bit cycle, a dynamic comparator detects the sign of the differential voltage on the summing nodes $\left(V_{X} P-V_{X N}\right)$ and dictates the polarity of the charge reference for use in the next cycle. To obviate the need for distributing an oversampled clock, self-timed SAR logic [33] is employed. By the end of the readout phase, a digital representation of the delayed-and-summed charge is available. To simplify the output routing, the differential outputs of the dynamic comparator (CPout+/-) are buffered and transmitted to the periphery of the ASIC, where the 10-bit parallel data are recovered and synchronized to a high-speed system clock for further processing.

Upon completion of a conversion, the summing nodes $\left(V_{X P}\right.$ and $\left.V_{X N}\right)$ are reset to prevent undesired signal attenuation associated with residue charge on the parasitic capacitors [1], as shown in Fig. 4(a) (CPRST). This operation also enables the calibration of the comparator offset in the background, as will be discussed in Section III. 


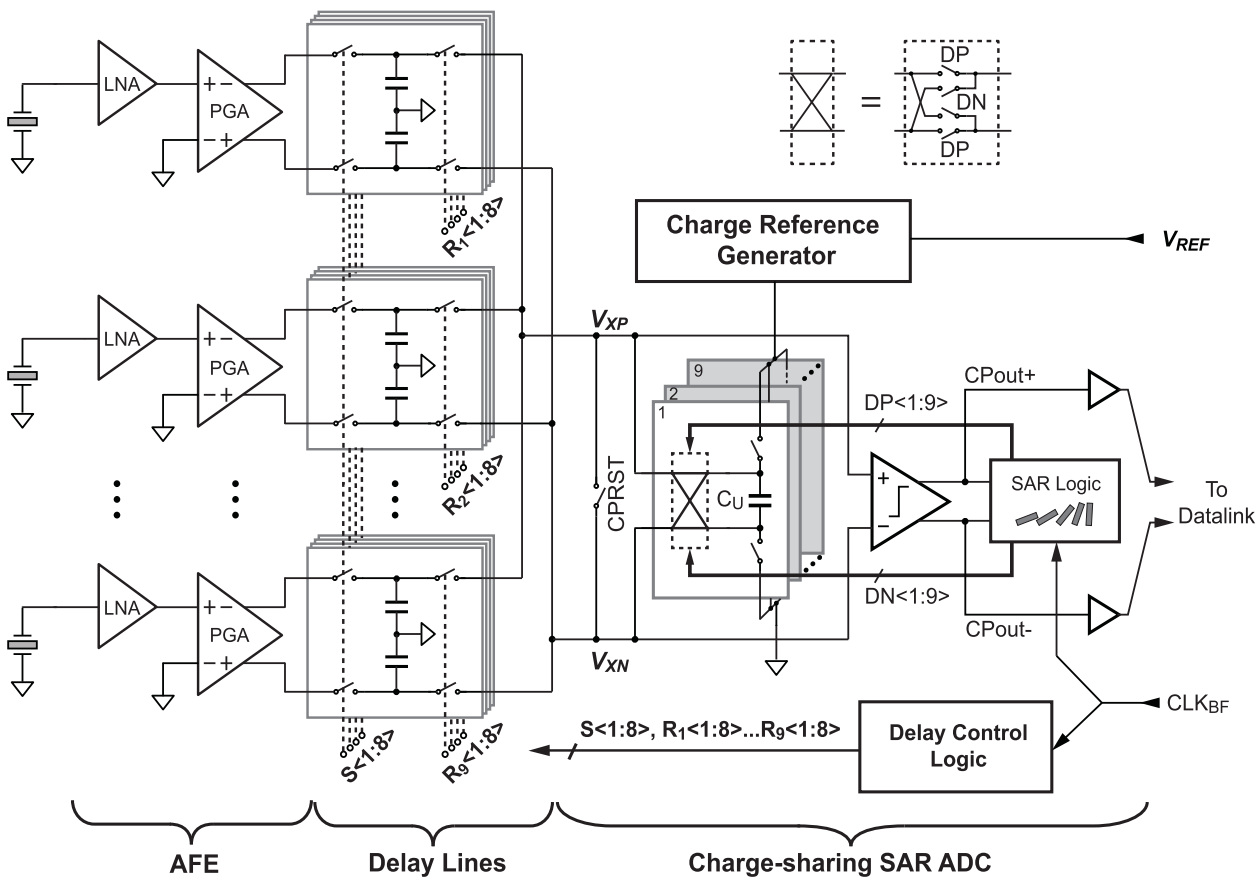

(a)

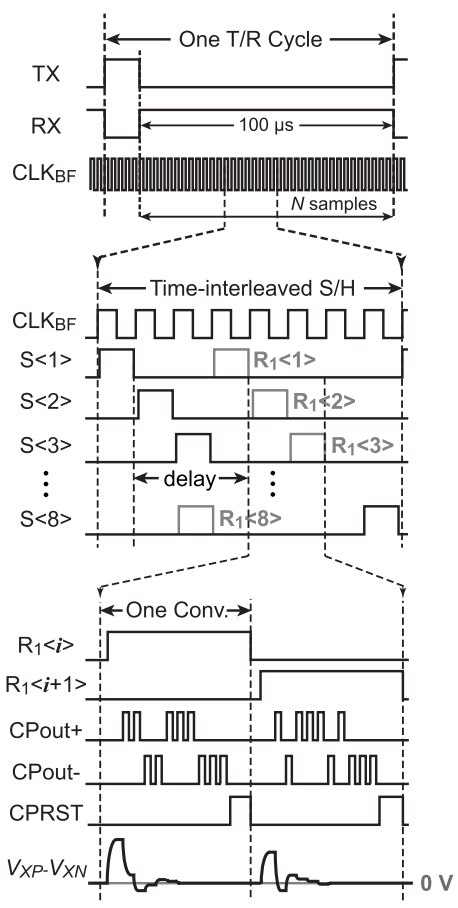

(b)

Fig. 4. (a) Schematic of the proposed beamforming ADC. (b) Associated operational timing diagram.

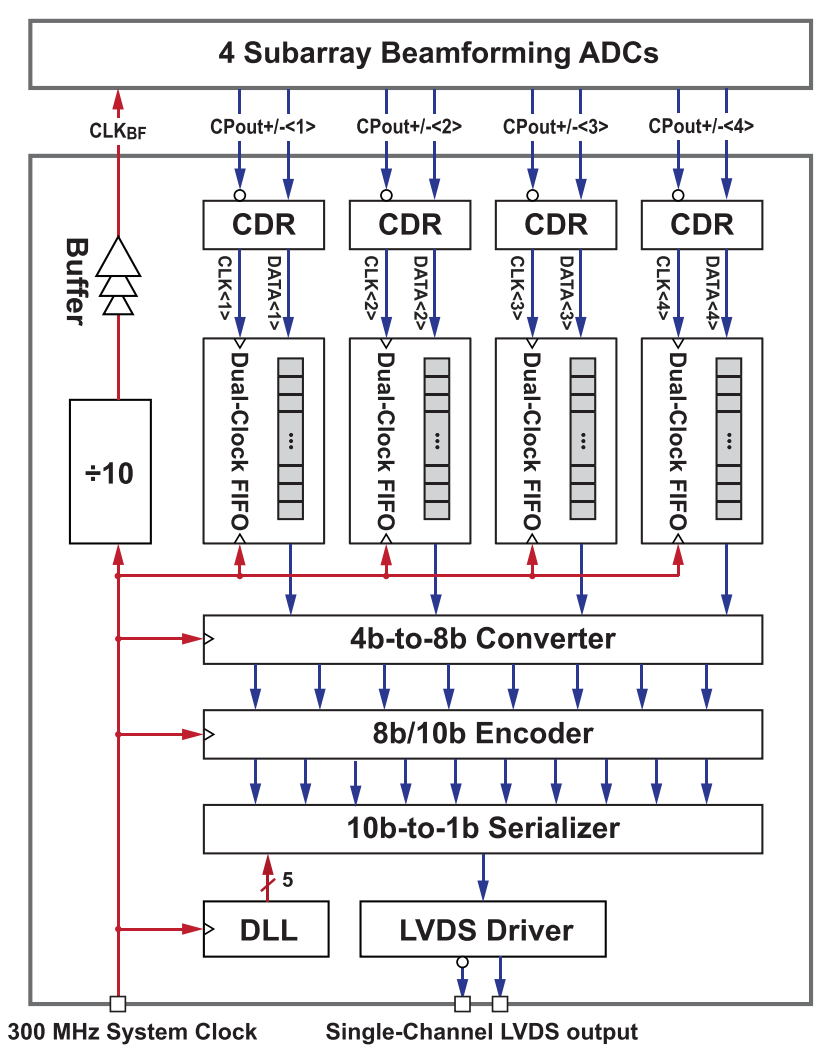

Fig. 5. Datalink architecture.

\section{Datalink}

Fig. 5 shows a block diagram of the datalink. Each subarray ADC is interfaced with a clock-data-recovery (CDR) circuit.
It converts the differential return-to-zero (RZ) outputs of the SAR comparator to a single-bit non-return-to-zero (NRZ) data stream, and extracts an asynchronous clock that is aligned with the recovered data. The data stream is then synchronized to a $300-\mathrm{MHz}$ global clock in an FIFO for further processing.

Before the serialization, every two consecutive 4-bit-wide FIFO outputs are concatenated to an 8 -bit byte by a $4 \mathrm{~b}-$ to- $8 \mathrm{~b}$ converter, which is then mapped to a 10-bit code in an $8 b / 10 b$ encoder [34]. This coding scheme facilitates clock recovery at the system side without relying on a per-channel clock line. Moreover, it ensures a DC-balance in the data stream, which helps both data recovery and error detection at the system side [34]. The 10-bit data are then serialized to a single-bit data stream at $1.5 \mathrm{~Gb} / \mathrm{s}$, which is buffered by an LVDS driver and transmitted over a twin-axial cable to the imaging system.

The ASIC receives a $300-\mathrm{MHz}$ global LVDS clock from the system, which is converted to CMOS logic levels at the periphery of the ASIC. Here, it serves as the main clock for the core of the datalink, and is multiplied by 5 in a delaylocked-loop (DLL) to generate the clock phases needed for serialization. It is divided down by 10 to produce the $30-\mathrm{MHz}$ beamforming clock $\left(\mathrm{CLK}_{\mathrm{BF}}\right)$, which is distributed across the subarray receivers via a balanced clock tree.

\section{Circuit Implementation}

\section{A. AFE}

The LNA in each AFE channel is an improved version of the design described in [1] and [35]. It is implemented as a single-ended capacitive feedback current-reuse amplifier with a split capacitor network to achieve a compact layout, and consumes $75 \mu \mathrm{A}$. 


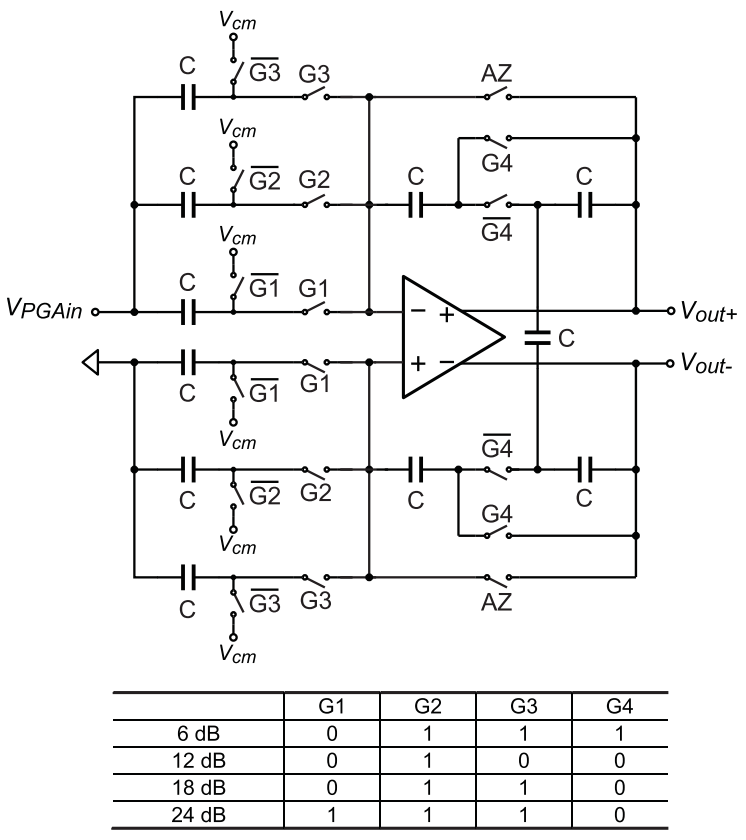

Fig. 6. Schematic of the PGA and the gain control code map.

The PGA implements three functions: 1) providing four fine gain steps to define the TGC gain resolution; 2) converting the LNA output to differential signals to drive the delay lines; 3 ) low-pass filtering prior to sampling to minimize aliasing. As shown in Fig. 6, a programmable capacitor network provides the desired gain levels ranging from 6 to $24 \mathrm{~dB}$ according to the control code map shown below. To save area, a T-type capacitor network [36], employing unit capacitors of $33 \mathrm{fF}$, is used as the feedback element across a compact differential telescopic amplifier. Each PGA consumes $100 \mu \mathrm{A}$.

The PGA drives a delay line that consists of eight stages of time-interleaved differential S/H memory cells. Each cell comprises a pair of grounded metal-insulator-metal (MIM) capacitors and a set of nMOS switches for sampling and readout. The capacitors are sized as large as possible (133 fF) within the available area to minimize the $k T / C$ noise contribution to the front end. The worst-case settling time constant of approximately $1.5 \mathrm{~ns}$ is less than $1 / 20$ of the sampling period, adequate for the required linearity in spite of the signal-dependent ON-resistance of the switches.

As discussed in [1], the mismatch of the S/H memory cells would introduce a ripple pattern with a period of $M / f_{S}$, where $M=8$ is the number of delay steps and $f_{S}$ is the sampling frequency. To mitigate this interference, the mismatchscrambling technique proposed in [1] is equally applicable to this work. As an alternative, however, since the beamformer outputs are digitized synchronously to the same system clock that controls the beamformer, the ripple patterns at different delay settings are pre-recorded and stored in memory, and then subtracted from the outputs during the normal receive phase. This calibration process is realized off-chip in the back-end digital processing unit (FPGA). This approach takes advantage of the integrated beamforming ADC, and thus not only saves area but also prevents adding the excess noise associated

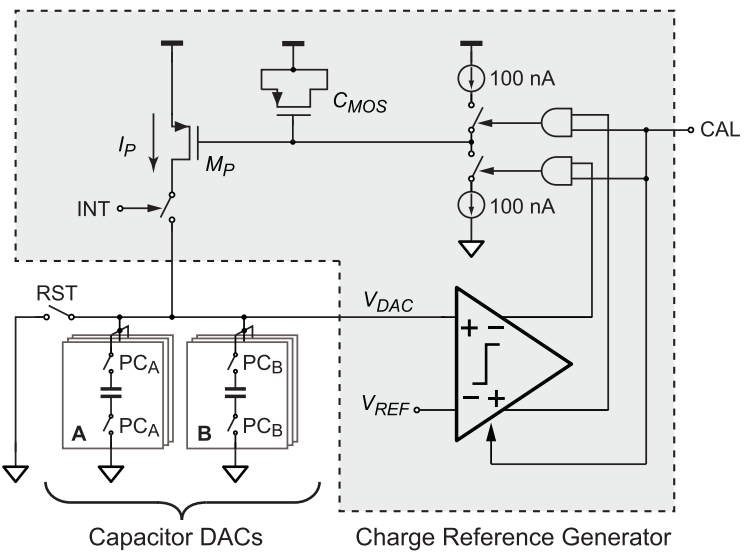

(a)

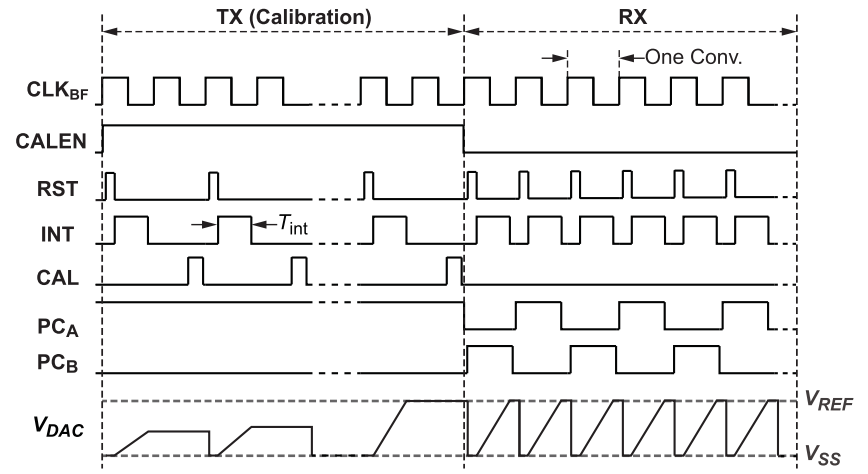

(b)

Fig. 7. (a) Schematic of the charge reference generator and (b) its timing diagram.

with the mismatch-scrambling technique [1], at the cost of an increased complexity in the back-end signal processing. Such extra complexity is modest, as only a relatively small set of ripple patterns need to be recorded, given that only a modest number of pre-steering directions are required, e.g., 25 in [25].

\section{B. Charge-Reference Generation}

The generation and distribution of references for a massively parallel ADC array is challenging. In prior implementations of charge-sharing SAR ADCs [32], [37], the CDAC is precharged by an external voltage source before the start of each conversion. Due to the significant load that this source needs to drive in a nanosecond time slot, this approach is prone to suffer from errors due to $d i / d t$ transients caused by the bondwire inductance, unless large on-chip decoupling capacitors are used. The alternative of employing on-chip reference buffers would introduce a significant power overhead [38].

In this work, we propose to precharge the CDAC with current sources to relax the power and area requirements for charge reference generation. Unlike the approach described in [38], the precharging current is locally generated in each subarray. To mitigate mismatch and PVT sensitivity, it is self-calibrated during the TX phase in reference to an external voltage $\left(V_{R E F}\right)$. This simplifies the system-level layout, as no global current reference distribution or high-current voltage reference routing is required.

Fig. 7 shows the schematic of the charge reference generator and its timing details. It consists of a gated current 
source $\left(M_{P}\right)$, a charge pump, and a calibration comparator. Intuitively, the charge reference generated by a gated current source can be written as

$$
Q_{R E F}=I_{P} \times T_{\text {int }}
$$

where $I_{P}$ is the precharging current and $T_{\text {int }}$ is the precharging duration defined on-chip. It is, however, difficult to maintain uniformity of $Q_{R E F}$ across the whole array, since both $I_{P}$ and $T_{\text {int }}$ are subject to process variations and mismatch. Therefore, we define the charge reference as

$$
Q_{R E F}=C_{D A C} \times V_{R E F}
$$

where $C_{D A C}$ is the total capacitance of the DAC array and $V_{R E F}$ is the desired voltage reference with respect to the AFE output.

As the absolute value of capacitors in modern CMOS processes is typically more strictly controlled [39], calibration is applied to $I_{P}$ so that the voltage on $C_{D A C}\left(V_{D A C}\right)$ after precharging approaches $V_{R E F}$. This is accomplished by introducing a calibration phase in synchronization with the TX phase, when digitization is not required. During this phase, the charge pump and the calibration comparator are periodically activated in a short-time interval (CAL) following each precharging cycle. The calibration comparator compares $V_{D A C}$ and $V_{R E F}$, and according to the result, a unit charge packet is pumped in or pulled out from a MOS memory capacitor $\left(C_{M O S}\right)$ at the gate of $M_{P}$ to adjust the value of $I_{P}$ for the next cycle. The size of the charge packet, which dictates the reference calibration resolution $\left(\mathrm{LSB}_{\mathrm{CAL}}\right)$, is set by both the pulse duration time and the magnitude of the sourcing/sinking current in the charge pump. This process repeats for a defined number of cycles, at the end of which $V_{D A C}$ has converged to $V_{R E F}$ to within $\pm 1 \mathrm{LSB}_{\mathrm{CAL}}$.

During the RX phase, both the charge pump and the calibration comparator are disabled, and the gated current source precharges $C_{D A C}$ based on the bias voltage stored on $C_{M O S}$. The leakage on $C_{M O S}(\sim 4 \mathrm{pF})$ would lead to a worst-case gain drift of $0.3 \mathrm{~dB}$ within one RX cycle $(100 \mu \mathrm{s})$, which is negligible given the significant time-dependent attenuation of ultrasound. The broadband white noise of the charge pump is sampled on $C_{M O S}$ and held constant throughout the RX phase, and therefore does not contribute any in-band noise.

Both the precharging current noise and the jitter of $T_{\text {int }}$ lead to noise charge sampled on $C_{D A C}$. The former is minimized by appropriate sizing of transistor $M_{P}\left(W_{P} / L_{P} \ll 1\right)$, leaving jitter of the precharging clock as the dominant noise source. This clock is derived from the ASIC input clock, whose jitter performance is, therefore, crucial. Maximizing $T_{\text {int }}$ helps in relaxing the jitter requirements. To do so, we use a ping-pong charge reference that consists of two identical CDAC arrays, as shown in Fig. 7. A duration time of $25 \mathrm{~ns}$ (3/4 of the sampling clock period) is allocated for $T_{\text {int }}$, permitting the use of a system clock with moderate jitter ( $\sim 20 \mathrm{ps})$.

In the calibration phase, only one DAC array is connected to the gated current source, while in the RX phase they are alternately used for precharging and conversions.

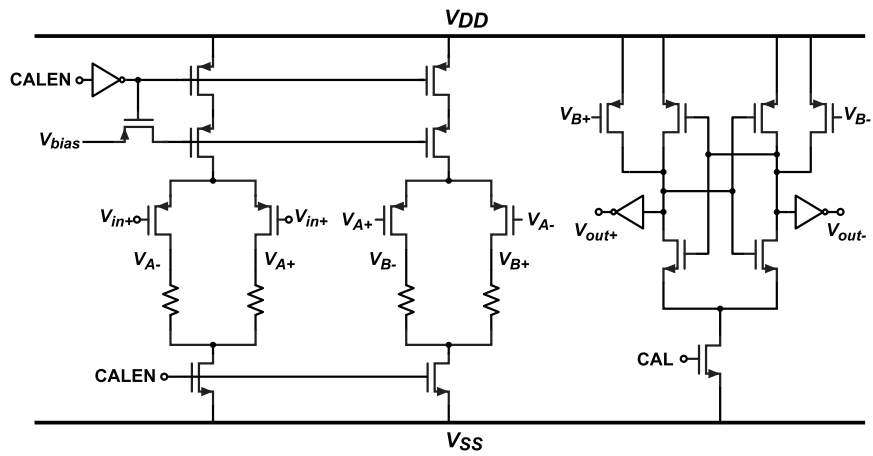

Fig. 8. Calibration comparator for charge-reference generation.

By sharing the current source for precharging and the timing logic for generating $T_{\text {int }}$, the ping-pong charge reference is free from interleaving spurs caused by the DAC capacitance mismatch.

The topology of each DAC array is similar to [37]. The charge references corresponding to the first 7 MSBs are produced by precharging a bank of binary-scaled capacitors, while those corresponding to the last $3 \mathrm{LSBs}$ are generated using charge redistribution. Metal-oxide-metal (MOM) capacitors of $23 \mathrm{fF}$ with symmetric plate parasitic capacitance are utilized as unit capacitors to ensure adequate matching for a 10-bit linearity. In total, 67 unit capacitors are used in each DAC, leading to a total capacitance of about $1.5 \mathrm{pF}$.

The required reference voltage is determined by the following charge-balancing equation:

$$
N \cdot V_{\mathrm{in}, \max } \cdot C_{M}=4 \cdot V_{R E F} \cdot C_{D A C}
$$

where $N$ is the number of subarray elements, $V_{\text {in,max }}$ is the maximum peak-to-peak differential PGA output swing, and $C_{M}$ is the capacitance of a unit memory cell in a delay line of the beamformer. The factor of 4 comes from the single-ended-to-differential conversion of $C_{D A C}$. For $N=9$, $V_{\text {in, } \max }=800 \mathrm{mV}, C_{M}=133 \mathrm{fF}$, and $C_{D A C} \approx 1.5 \mathrm{pF}, V_{R E F}$ is approximately $160 \mathrm{mV}$.

The calibration comparator is implemented as a StrongArm latch following a class-A preamplifier that is only powered on when CALEN is high (Fig. 8). The preamplifier consists of two cascaded stages of resistively loaded differential pairs to warrant a sufficient gain. The first stage is dimensioned to minimize both noise and offset. Given the relatively low reference voltage, a pMOS input pair is used. Since the comparator is powered down during the RX phase, its contribution to the overall power consumption is negligible.

\section{SAR Logic}

For an implementation in $0.18-\mu \mathrm{m}$ CMOS, the high-speed SAR logic readily dominates the power consumption of the ADC, and therefore needs to be carefully minimized. Fig. 9 shows the schematic and the timing diagram of the proposed asynchronous SAR logic. The differential outputs of the dynamic comparator (CPout $+/-$ ) directly trigger a 10 -stage differential shift-register, where each stage consists of a pair of D-flip-flops (DFFs) with an enable input. Compared to 


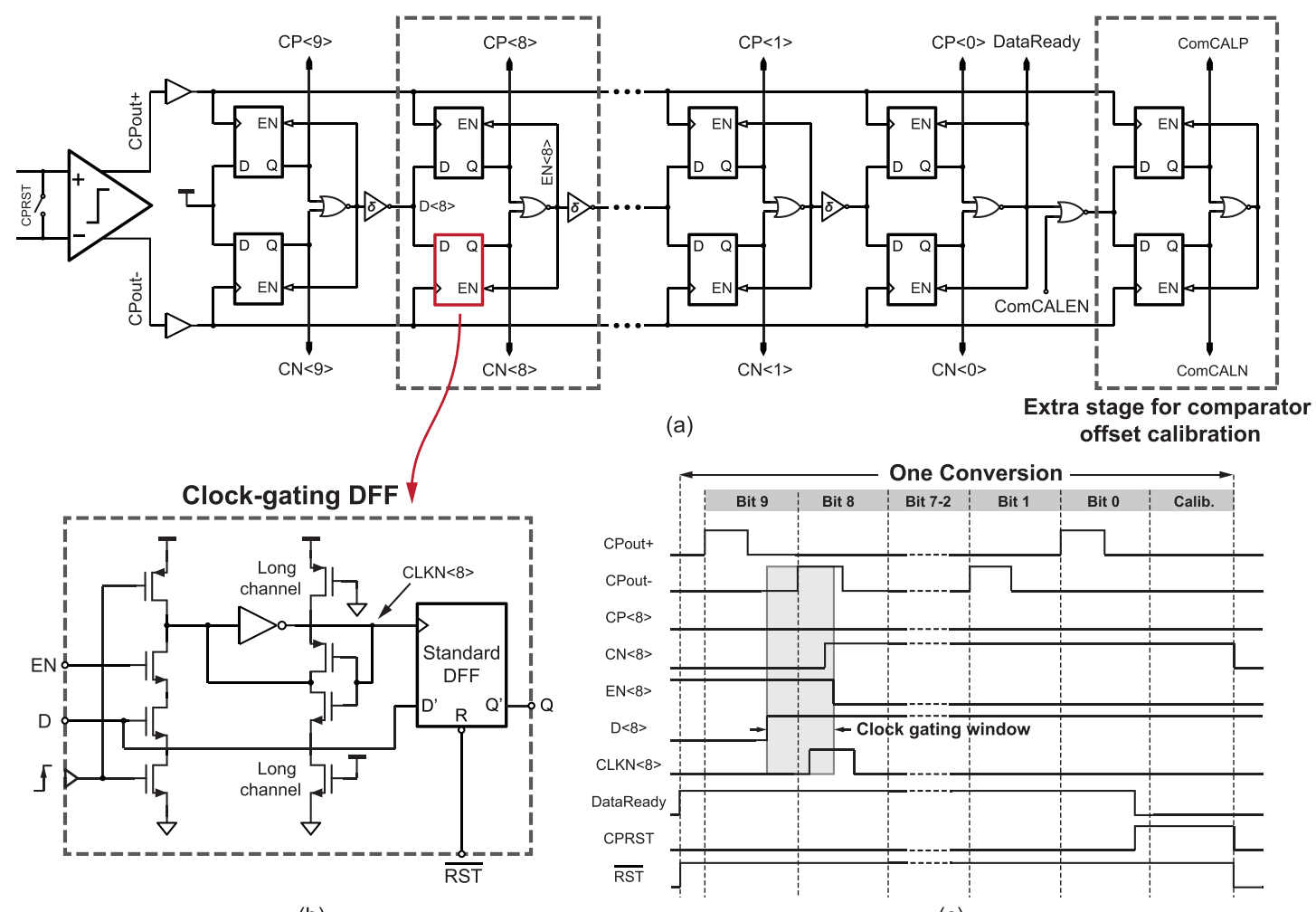

(b)

(c)

Fig. 9. (a) Schematic of the proposed asynchronous SAR logic. (b) Clock-gating DFF. (c) Timing diagram in each conversion.

conventional implementations [29], [30], the proposed scheme minimizes the time delay between the comparator decision and the DAC switching, thus relaxing the timing for charge sharing. During the conversion, each DFF pair reads the comparator decision by sensing the rising edges of the comparator outputs. Once a rising edge on either side is detected, the DFF pair is immediately disabled and no longer responsive to succeeding comparison events until the end of the cycle. The data are then captured and frozen to control the switching of corresponding DAC elements. To identify the completion of a conversion, the enable signal of the LSB DFF is used as the DataReady signal. An additional DFF pair is used for comparator offset calibration, as will be discussed in Section III-D.

To further reduce the dynamic power consumption, each DFF pair is kept deactivated until the preceding stage has come to a decision. Thus, in every bit cycle only one DFF pair is clocked for data reading. This is achieved by embedding a local clock-gating buffer within each DFF cell, which defines a bit-wise timing window based on the outputs of the previous and the present stages [Fig. 9(c)]. The clock-gating buffer is implemented as a dynamic NAND gate followed by a simple latch with a weak feedback inverter [40] [Fig. 9(b)]. To prevent undesired switching events, the output of the previous stage is delayed to guarantee that its rising edge always arrives during the reset phase of the dynamic comparator. Simulation results indicate a 33\% dynamic power reduction in the SAR logic thanks to the adoption of the clock-gating scheme.

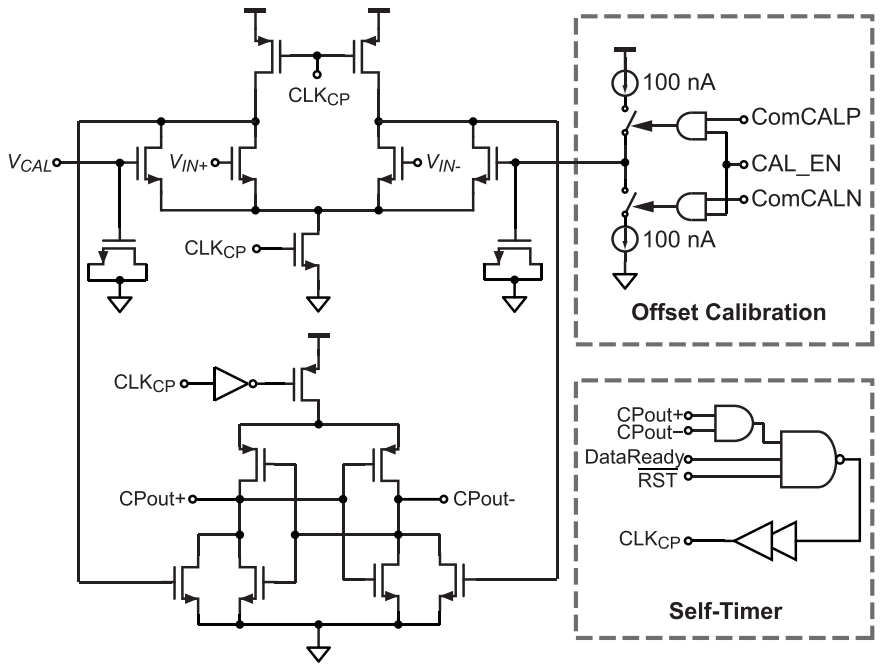

Fig. 10. Dynamic comparator with offset calibration.

\section{Dynamic Comparator}

An inherent limitation of the charge-sharing SAR conversion is the discrepancy between the charge-domain signal approximation and the voltage-domain quantization, which leads to more stringent requirements on the input-referred noise and offset of the dynamic comparator [37]. Fig. 10 shows the schematic of the dynamic comparator, the core of which is a double-rail latch-type voltage sense amplifier [41]. Its first stage is sized to ensure a sufficiently low input-referred noise. 


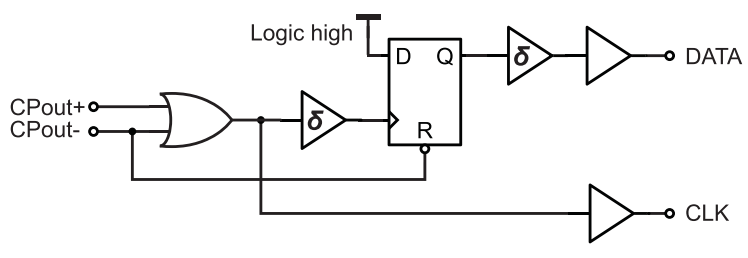

Fig. 11. Schematic of the CDR circuit.

A self-timer circuit takes the comparator outputs and the DataReady signal to generate an oversampling clock that schedules the evaluation and reset of the dynamic comparator.

In contrast to a voltage-domain SAR ADC, the input offset of the comparator in a charge-sharing ADC would result in a dynamic charge offset that degrades the conversion linearity [37], and therefore should be minimized. Such offset is dependent on the input common-mode voltage [30] during charge sharing, which, in turn, depends on the parasitic capacitance at the summing nodes and therefore varies between subarrays. To avoid the need for individual offset trimming for each subarray, the offset is self-calibrated in a way similar to [42], which involves a charge pump and an auxiliary input pair with one gate connecting to an external calibration voltage. In contrast with [42], the offset calibration is performed in the background while the SAR conversion is on-going. As described in Section II-C, the comparator input nodes are shorted to clear the residual charge at the end of each conversion, resulting in an input voltage that is close to the common-mode voltage during the LSB cycles. Therefore, by triggering one more comparison, the polarity of the offset can be detected, allowing the charge pump to adjust the bias voltage of the auxiliary input pair. This additional comparison is realized by adding an extra stage in the asynchronous SAR logic, as shown in Fig. 9. By repeating this process for successive SAR conversions, the offset voltage is progressively minimized within a finite number of ADC cycles. The background calibration can be disabled by nulling the input of the extra logic stage.

Because of the common-mode charge stored on the parasitic capacitances of the CDAC and switches during precharging, as well as the relatively low reference voltage, the input common mode of the comparator slightly decreases as the SAR conversion proceeds, which leads to a bit-dependent dynamic offset. However, since the variation of the input common mode is only a small portion of its absolute value, the resulting offset variation and dynamic error charge have a negligible impact on the linearity.

\section{E. CDR and FIFO}

The differential comparator outputs from each subarray are received by CDR circuits at the ASIC periphery, which reconstruct the serial ADC output and a corresponding asynchronous clock (Fig. 11). Since the comparator outputs are in RZ format, the clock can be reconstructed from an "OR" operation of the two outputs. A DFF driven by this clock reconstructs the serial ADC data. The DFF has a constant logic high input and is reset by the negative comparator

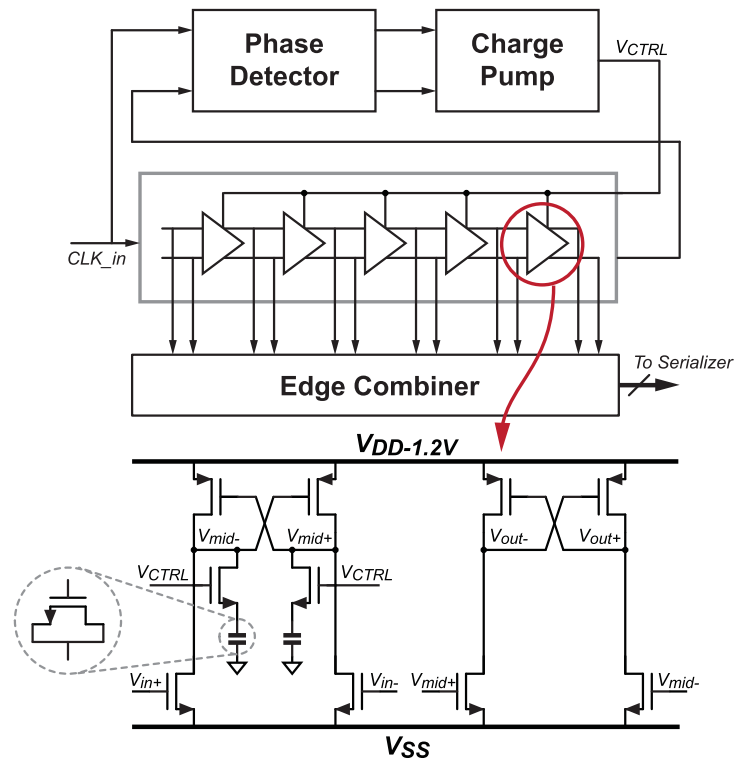

Fig. 12. Schematic of the DLL.

output (CPout-). Proper delays are added to enhance the circuit's immunity to timing uncertainties.

The recovered clock and data are fed to a dual-clock FIFO for further synchronization. The "read" operation of the FIFO is driven by the $300-\mathrm{MHz}$ global clock. In order to simplify the data reconstruction at the system side, once a valid data stream is received, the FIFO is expected to operate in neither "empty" state nor "full" state. The "full" state is avoided by selecting an FIFO queue-depth of 16, more than the 10 bits of a single conversion result. To avoid the "empty" state, a five-cycle delay is applied between the start of the "write" operation and the start of the "read" operation to make sure that enough data are written to the FIFO before reading. The FIFO and the following encoders were implemented and optimized using logic synthesis tools. To enable a bit-error-rate (BER) test for the high-speed data exportation, the FIFO inputs can be switched to the output of an on-chip pseudo-random bit sequence (PRBS) generator with a sequence length of $2^{16}-1$.

\section{F. $D L L$}

Fig. 12 shows a block diagram of the DLL, which is based on [43]. It consists of a five-stage differential voltagecontrolled delay line (VCDL), a phase detector, a charge pump, and an edge combiner. The delay cell in the VCDL is implemented by cascading two differential cross-coupled inverters, the first of which is loaded by $R C$ branches consisting an nMOS switch and a MOS capacitor. By increasing the switch control voltage $V_{C T R L}$, more capacitance is added to the inverter's load, thus increasing the delay. Once the loop is stable, the edge combiner receives the outputs of the delay cells and generates five consecutive equal-width pulses, which are fed into a 10:1 multiplexer for data serialization.

\section{EXPERIMENTAL RESULTS}

The ASIC has been fabricated in a $0.18-\mu \mathrm{m} 1 \mathrm{P} 6 \mathrm{M}$ lowvoltage CMOS process and has an area of $4.8 \times 2.5 \mathrm{~mm}^{2}$, 


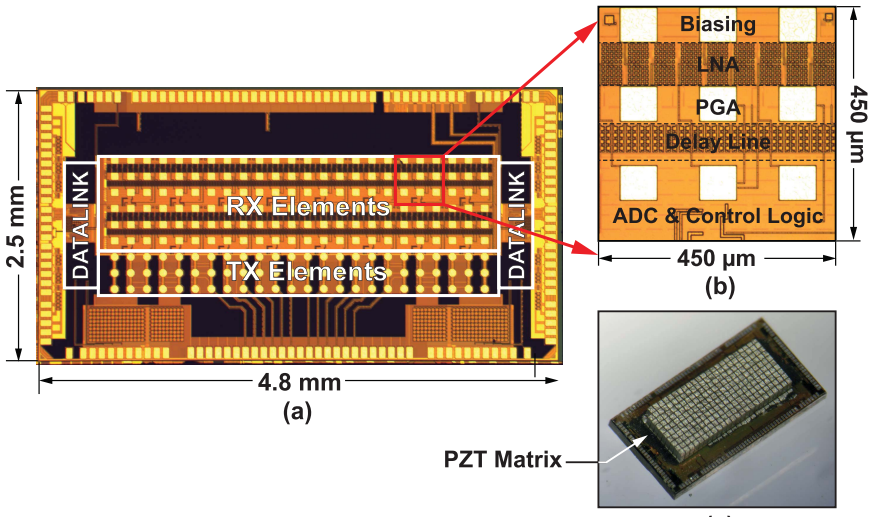

(c)

Fig. 13. (a) Microphotograph of the ASIC. (b) Floorplan of one $3 \times 3$ subarray receiver. (c) A fabricated prototype with integrated PZT matrix transducer.

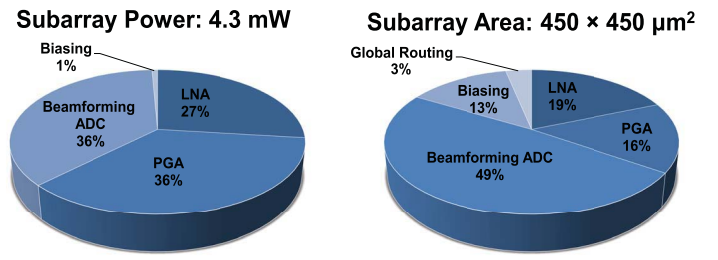

Fig. 14. Power and area breakdown of one $3 \times 3$ subarray receiver.

as shown in Fig. 13(a). The floor plan of a $3 \times 3$ subarray receiver is shown in Fig. 13(b), while its power and area breakdown are shown in Fig. 14. The bondpads for transducer interconnection are implemented in the top (6th) metal layer, while the 5th metal layer is reserved as a grounded shield to protect the LNA inputs from digital interference. All building blocks are powered by a $1.8-\mathrm{V}$ supply except for the VCDL in the DLL, which is powered by a separate $1.2-\mathrm{V}$ supply. While receiving, each subarray receiver consumes $4.3 \mathrm{~mW}$, corresponding to $0.46 \mathrm{~mW} /$ element. The beamforming ADC along with the delay programming logic occupies about half of the subarray area, while consuming only $36 \%$ of the subarray power $(1.58 \mathrm{~mW})$. The total power including the datalink and LVDS drivers is $130.5 \mathrm{~mW}$, corresponding to $0.91 \mathrm{~mW} / \mathrm{element}$.

Fig. 13(c) shows a fabricated prototype with an integrated $24 \times 9$ PZT matrix transducer. It is wire-bonded to a daughter PCB for both electrical and acoustic tests. The daughter PCB is mounted on a custom-designed mother PCB, where an FPGA receives and buffers the high-speed RF data before transmitting it to a $\mathrm{PC}$ for image reconstruction.

\section{A. Electrical Measurements}

The electrical performance of the prototype ASIC has been characterized by wire-bonding test input signals to the selected RX transducer pads. The reconstructed digital outputs of each subarray receiver are converted back to a voltage signal according to (3) to facilitate the performance evaluation.

Fig. 15 shows the measured subarray receiver transfer function at 12 AFE gain settings. It achieves an overall mid-band

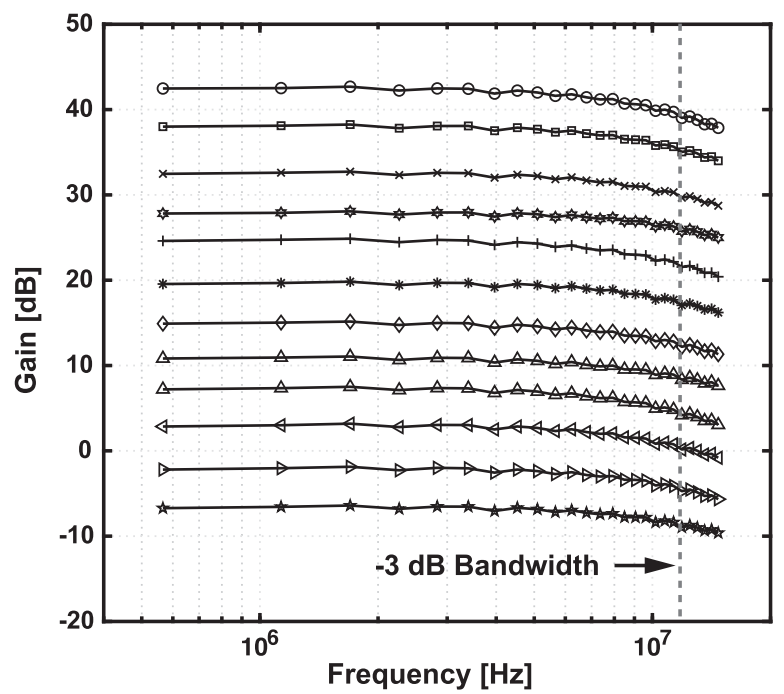

Fig. 15. Measured RX transfer function at 12 AFE gain settings.

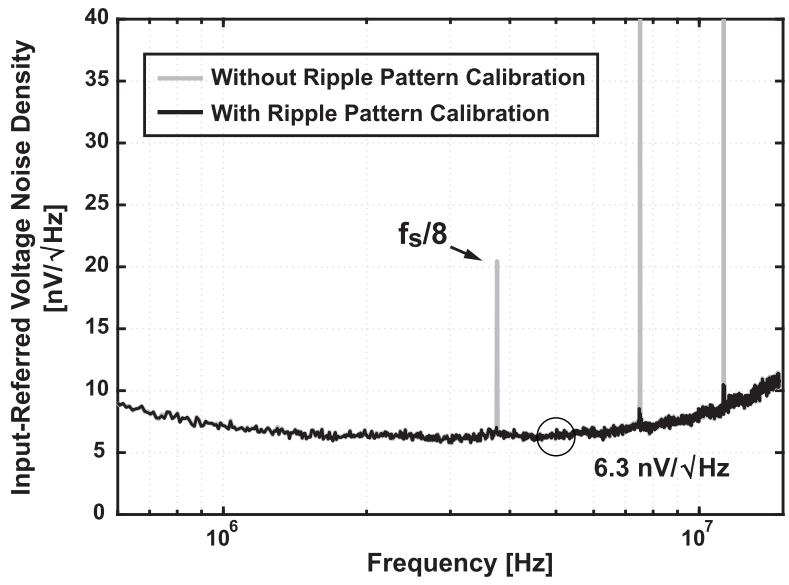

Fig. 16. Measured input-referred noise density spectrum of one subarray receiver.

gain range of $49 \mathrm{~dB}$, stepping from -7 to $42 \mathrm{~dB}$ with an average step size of $4.5 \mathrm{~dB}$. The deviation from the ideal gain step $(6 \mathrm{~dB})$ is mainly caused by the insufficient open-loop gain of the PGA core amplifier at high gain modes. The average $-3 \mathrm{~dB}$ bandwidth of the subarray receiver is measured as $11.9 \mathrm{MHz}$.

Fig. 16 shows the measured subarray input-referred voltage noise spectrum at the highest AFE gain setting, which indicates an input-referred voltage noise density of $6.3 \mathrm{nV} / \sqrt{ } \mathrm{Hz}$ at $5 \mathrm{MHz}$. Before applying the digital back-end calibration (Section III-A), the ripple pattern introduced by delay-line mismatches appears as in-band interference tones at $f_{S} / 8$ $(3.75 \mathrm{MHz})$ and its harmonics. By subtracting the pre-recorded ripple pattern (obtained from 100 iterations with grounded inputs) from the output signal, these interference tones get significantly reduced from the spectrum without deteriorating the noise floor.

Fig. 17 shows the measured output spectrum of one subarray receiver at the highest AFE gain setting with a $4.95-\mathrm{MHz}$ sinusoidal test input with a peak value of $3.1 \mathrm{mV}$. 
TABLE I

COMPARISON OF THE STATE-OF-THE-ART UltRASOUND Digitization SOLUTIONS

\begin{tabular}{|c|c|c|c|c|c|c|}
\hline & This Work & {$[16]$} & [17] & {$[18]$} & [44] & [1] \\
\hline Process & $180 \mathrm{~nm}$ & $130 \mathrm{~nm}$ & $28 \mathrm{~nm}$ & $130 \mathrm{~nm}$ & $180 \mathrm{~nm}$ & $180 \mathrm{~nm}$ \\
\hline Transducer & PZT & CMUT & CMUT & N/A & N/A & PZT \\
\hline RX Array Size & $24 \times 6$ & $64 \times 1$ & $4 \times 4$ & N/A & N/A & $32 \times 32$ \\
\hline No. of Channels & 144 & 64 & 16 & 64 & 128 & 864 \\
\hline Center Freq. & $5 \mathrm{MHz}$ & $3 \mathrm{MHz}$ & $5 \mathrm{MHz}$ & $5 \mathrm{MHz}$ & $2.5-4.5 \mathrm{MHz}$ & $5 \mathrm{MHz}$ \\
\hline Output Format & Digital & Digital & Digital & Digital & Digital & Analog \\
\hline RX Architecture & $\begin{array}{l}\text { AFE+Analog S/H } \\
+ \text { ADC + Datalink }\end{array}$ & $\begin{array}{l}\text { Analog S/H + } \\
\text { ADC + FIFO }\end{array}$ & $\begin{array}{c}\mathrm{AFE}+\mathrm{ADC}+ \\
\text { FIFO }\end{array}$ & $\mathrm{ADC}+\mathrm{FIFO}$ & $\begin{array}{l}\text { AFE+ADC } \\
+ \text { Datalink }\end{array}$ & $\begin{array}{c}\text { AFE }+ \\
\text { Analog S/H }\end{array}$ \\
\hline ADC Architecture & SAR & SAR & $\Delta \Sigma$ & SAR & $\Delta \Sigma$ & $\mathrm{N} / \mathrm{A}$ \\
\hline Nyquist Rate & $30 \mathrm{MHz}$ & $20-40 \mathrm{MHz}$ & $20 \mathrm{MHz}$ & $20-40 \mathrm{MHz}$ & $20 \mathrm{MHz}$ & $33 \mathrm{MHz}$ \\
\hline Pitched-Matched & Yes $(150 \mu \mathrm{m})$ & No & Yes $(250 \mu \mathrm{m})$ & No & No & Yes $(150 \mu \mathrm{m})$ \\
\hline Channel Reduction & 36-fold & 64-fold & 16-fold & 64-fold & 2-fold & 9-fold \\
\hline $\begin{array}{c}\text { Transducer } \\
\text { Integration Method }\end{array}$ & Direct Integration & N/A & Flip-chip & N/A & N/A & Direct Integration \\
\hline Delay Resolution & $33 \mathrm{~ns}$ & $6.25 \mathrm{~ns}$ & $8.33 \mathrm{~ns}$ & $6.25 \mathrm{~ns}$ & N/A & $30 \mathrm{~ns}$ \\
\hline Active Area/el. & $0.026 \mathrm{~mm}^{2 *}$ & $0.303 \mathrm{~mm}^{2}$ & $0.088 \mathrm{~mm}^{2}$ & $0.473 \mathrm{~mm}^{2}$ & $\mathrm{~N} / \mathrm{A}$ & $0.023 \mathrm{~mm}^{2}$ \\
\hline RX Power/el. & $0.91 \mathrm{~mW}^{*}$ & $17.81 \mathrm{~mW}$ & $33.19 \mathrm{~mW}$ & $9.45 \mathrm{~mW}$ & $36.1 \mathrm{~mW}$ & $0.27 \mathrm{~mW}$ \\
\hline Beamforming Gain & $9.5 \mathrm{~dB}$ & $18 \mathrm{~dB}$ & $0 \mathrm{~dB}$ & $18 \mathrm{~dB}$ & $0 \mathrm{~dB}$ & $9.5 \mathrm{~dB}$ \\
\hline Peak SNDR & $51.8 \mathrm{~dB}$ & N/A & $58.9 \mathrm{~dB}^{* *}$ & $48.5 \mathrm{~dB}$ & $65 \mathrm{~dB}$ & $51 \mathrm{~dB}^{* * *}$ \\
\hline
\end{tabular}

${ }^{*}$ Including the datalink and LVDS drivers. $\quad{ }^{* *} \mathrm{ADC}$ only, excluding the analog front-end.

${ }^{* * *}$ Calculated as signal power/noise power, measured without mismatching scrambling.

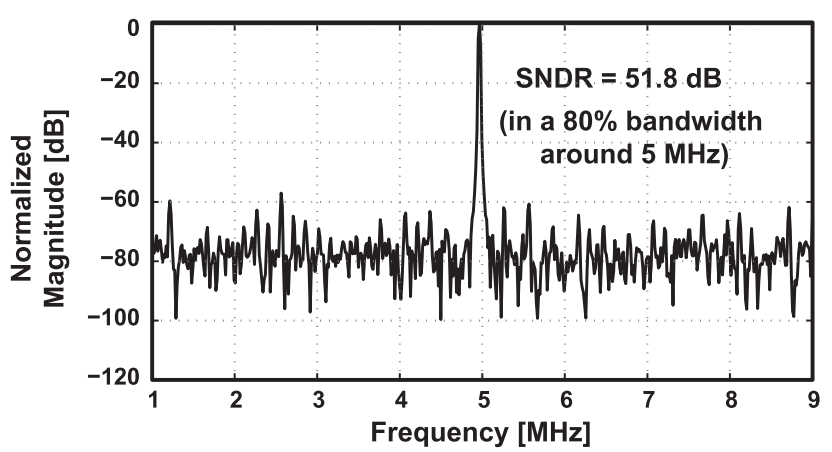

Fig. 17. Measured RX subarray output spectrum with a sinusoidal test input.

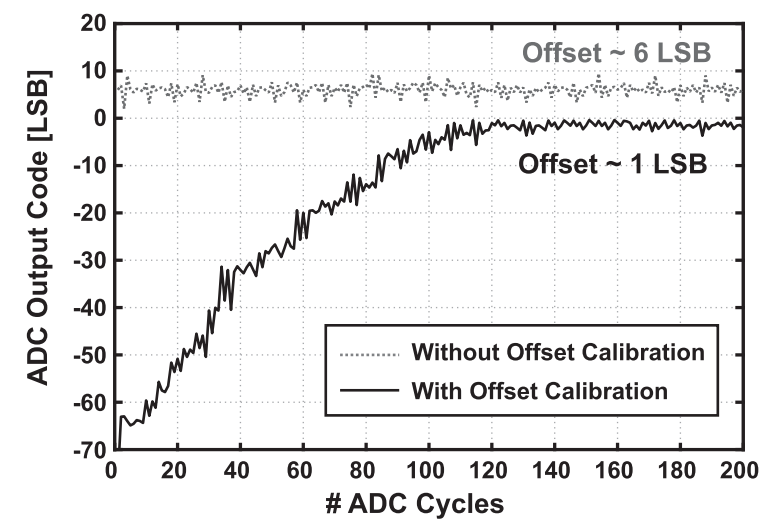

Fig. 18. Convergence of the background comparator offset calibration process.

It achieves a peak SNDR of $51.8 \mathrm{~dB}$ within an $80 \%$ bandwidth (3-7 MHz) around the center frequency $(5 \mathrm{MHz})$, where the AFE dominates the noise floor.

Fig. 18 shows the transient response of one subarray output with the proposed background comparator offset calibration enabled. Upon initialization, the offset settling process takes about 120 ADC cycles $(\sim 4 \mu \mathrm{s})$ to converge. Without calibration, the original output offset of the tested subarray (also

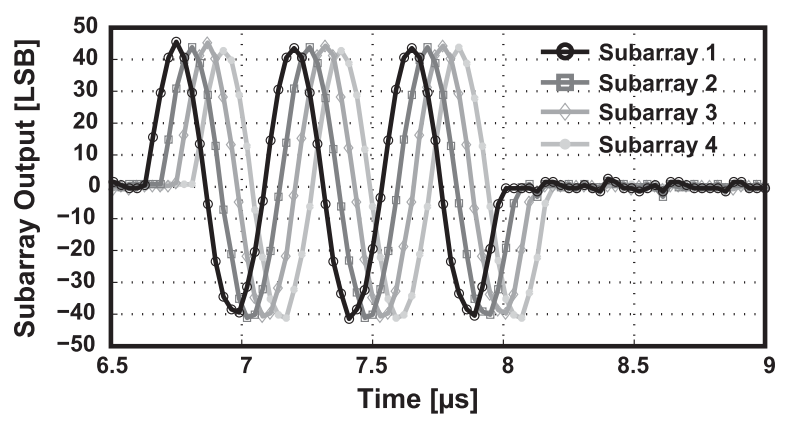

Fig. 19. Reconstructed four-subarray outputs from a single LVDS channel.

shown in Fig. 18) is about $6 \mathrm{LSB}$, which is reduced to $-1 \mathrm{LSB}$ after calibration.

The high-speed datalink has been evaluated separately using the on-chip PRBS generator, which shows a BER better than $10^{-9}$ across $1-\mathrm{m}$ coaxial cables. To better demonstrate the channel-reduction capability of the datalink, we programmed four subarrays that share the same high-speed data output channel with differential uniform delays $(33,100,166$, and $233 \mathrm{~ns}$ ). A three-cycle sinusoidal signal is chosen as the common input to these subarrays, with a frequency of $2 \mathrm{MHz}$ so as to better illustrate the relative time delay. Fig. 19 depicts the reconstructed time-domain output waveform of these four subarrays, recovered from the shared LVDS output port, which clearly shows the expected relative time delays. The worst-case inter-subarray crosstalk is measured as $-57 \mathrm{dBc}$. The measured gain mismatch across 16 subarrays is less than $0.1 \mathrm{~dB}$.

Table I illustrates the comparison of this paper with the state-of-the-art digitization solutions for 3-D ultrasound imaging systems [16]-[18], [44]. Based on Table I, this work achieves a $10 \times$ improvement in power efficiency, as well as a $3.3 \times$ improvement in integration density. When compared with our previous analog output receiver ASIC [1], the subarray digitization only costs about $70 \%$ extra power and is 


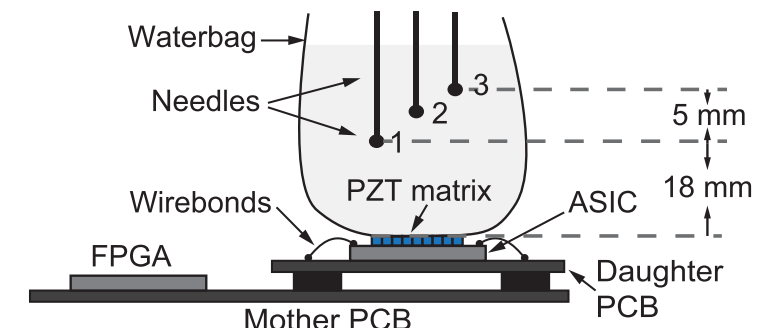

(a)

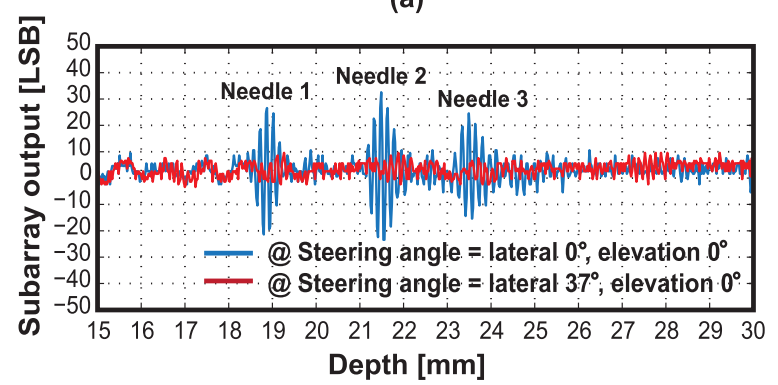

(b)

Fig. 20. (a) Setup for imaging experiments. (b) Recorded subarray output waveforms with different lateral steering angles.

realized within the same die size. On the other hand, the highspeed datalink introduces a non-negligible power overhead due to the relatively large feature size of the chosen technology. This, however, can be reduced in the future by adopting a more advanced CMOS technology, or applying on-chip digital beamforming [14] or compression techniques [15].

\section{B. Acoustic Measurements}

The acoustic performance of the fabricated prototype has been characterized by mounting a waterbag on the top of the PZT-on-ASIC assembly, as shown in Fig. 20(a). A three-needle phantom was immersed in water and placed at about $20 \mathrm{~mm}$ in front of the PZT matrix. A diverging wave was transmitted from the prototype by driving six elements at the center of the TX subarray (Fig. 2) using 20-V (peak-to-peak) three-cycle pulses. In several successive TX-RX cycles, the 16 subarrays in the prototype were steered to different angles to scan the volume.

Fig. 20(b) shows the recorded digital outputs of one subarray receiver with different programmed steering angles at the lateral direction. It clearly shows an increase of the echo amplitude when the subarray beamformer is steered toward the specific needle.

Fig. 21 illustrates a reconstructed B-mode image in the lateral direction. It is obtained by recording and combing the digital outputs of all subarrays, and performing the post-beamforming computation in software. The positions of all three needles are clearly shown in the image with a spatial resolution in line with the relatively small RX aperture.

The image was reconstructed from 25 beams (TX/RX cycles) with a pulse-repetition frequency of $5 \mathrm{kHz}$, leading to a theoretical volume rate of 200 volumes/s. In practice, however, the imaging rate is limited by the data transfer speed between the FPGA and the PC, as well as the software post-beamforming computing time. This constraint could be

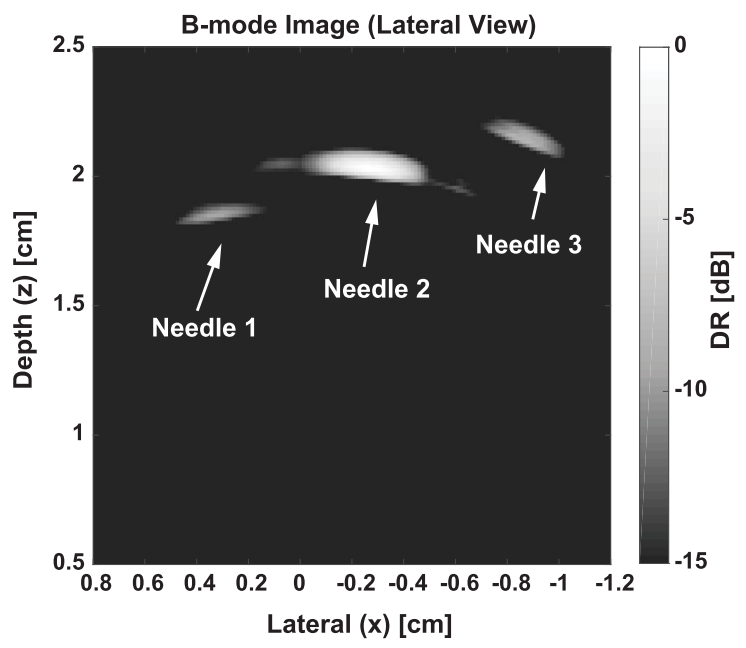

Fig. 21. Reconstructed B-mode image. The visible sidelobes of Needle 2 are as expected for the relatively small RX aperture.

resolved by migrating the image reconstruction function to the FPGA [45], or implementing it in a digital ASIC [14].

\section{CONCLUSION}

We have presented a front-end ASIC that enables powerand area-efficient in-probe digitization for the next-generation miniature 3-D ultrasound probes. It employs beamforming ADCs and high-speed datalinks to realize an additional 4-fold channel-count reduction compared to prior analog subarray beamformer designs. The ADC directly digitizes the subarray beamformer output in the charge domain to eliminate the need for intermediate power-hungry buffers. Self-calibrated charge references are proposed to further optimize the power efficiency as well as to facilitate the system-level design. The ASIC provides an overall 36-fold channel-count reduction and consumes less than $1 \mathrm{~mW} /$ element in receive, thus paving the way toward sub-Watt digital probes with $1000+$ elements. A prototype with integrated transducers has been successfully applied in a 3-D imaging experiment.

\section{REFERENCES}

[1] C. Chen et al., "A front-end ASIC with receive sub-array beamforming integrated with a $32 \times 32$ PZT matrix transducer for 3-D transesophageal echocardiography," IEEE J. Solid-State Circuits, vol. 52, no. 4, pp. 994-1006, Apr. 2017.

[2] D. Wildes et al., "4-D ICE: A 2-D array transducer with integrated asic in a 10-Fr catheter for real-time 3-D intracardiac echocardiography," IEEE Trans. Ultrason., Ferroelectr., Freq. Control, vol. 63, no. 12, pp. 2159-2173, Dec. 2016.

[3] M. Tan et al., "A front-end ASIC with high-voltage transmit switching and receive digitization for forward-looking intravascular ultrasound," in Proc. IEEE Custom Integr. Circuits Conf. (CICC), Austin, TX, USA, Apr. 2017, pp. 1-4.

[4] G. Gurun et al., "Single-chip CMUT-on-CMOS front-end system for real-time volumetric IVUS and ICE imaging," IEEE Trans. Ultrason., Ferroelectr., Freq. Control, vol. 61, no. 2, pp. 239-250, Feb. 2014.

[5] I. O. Wygant et al., "Integration of 2D CMUT arrays with front-end electronics for volumetric ultrasound imaging," IEEE Trans. Ultrason., Ferroelectr., Freq. Control, vol. 55, no. 2, pp. 327-342, Feb. 2008.

[6] C. Chen et al., "A prototype PZT matrix transducer with low-power integrated receive ASIC for 3-D transesophageal echocardiography," IEEE Trans. Ultrason., Ferroelectr., Freq. Control, vol. 63, no. 1, pp. 47-59, Jan. 2016. 
[7] B. Savord and R. Solomon, "Fully sampled matrix transducer for real time 3D ultrasonic imaging," in Proc. IEEE Ultrason. Symp., Oct. 2003, pp. 945-953.

[8] Z. Yu et al., "Front-end receiver electronics for a matrix transducer for 3-D transesophageal echocardiography," IEEE Trans. Ultrason., Ferroelectr, Freq. Control, vol. 59, no. 7, pp. 1500-1512, Jul. 2012.

[9] Y. Katsube et al., "Single-chip 3072ch 2D array IC with RX analog and all-digital TX beamformer for 3D ultrasound imaging," in Proc. IEEE Int. Solid-State Circuits Conf. (ISSCC) Dig. Tech. Papers, Feb. 2017, pp. $458-459$.

[10] C. Daft, S. Panda, P. Wagner, and I. Ladabaum, "Two approaches to electronically scanned 3D imaging using cMUTs," in Proc. IEEE Int. Ultrason. Symp. (IUS), Oct. 2006, pp. 685-688.

[11] T. M. Carpenter, M. W. Rashid, M. Ghovanloo, D. M. J. Cowell, S. Freear, and F. L. Degertekin, "Direct digital demultiplexing of analog TDM signals for cable reduction in ultrasound imaging catheters," IEEE Trans. Ultrason., Ferroelectr., Freq. Control, vol. 63, no. 8, pp. 1078-1085, Aug. 2016.

[12] Q. Liu, C. Chen, Z.-Y. Chang, C. Prins, and M. A. P. Pertijs, "A mixedsignal multiplexing system for cable-count reduction in ultrasound probes," in Proc. IEEE Int. Ultrason. Symp. (IUS), Oct. 2015, pp. 1-4.

[13] M. W. Rashid, C. Tekes, M. Ghovanloo, and F. L. Degertekin, "Design of frequency-division multiplexing front-end receiver electronics for CMUT-on-CMOS based intracardiac echocardiography," in Proc. IEEE Int. Ultrason. Symp. (IUS), Sep. 2014, pp. 1540-1543.

[14] B. Lam, M. Price, and A. P. Chandrakasan, "An ASIC for energyscalable, low-power digital ultrasound beamforming," in Proc. IEEE Int. Workshop Signal Process. Syst., Oct. 2016, pp. 57-62.

[15] T. Chernyakova and Y. Eldar, "Fourier-domain beamforming: The path to compressed ultrasound imaging," IEEE Trans. Ultrason., Ferroelectr., Freq. Control, vol. 61, no. 8, pp. 1252-1267, Aug. 2014.

[16] J.-Y. Um et al., "An analog-digital-hybrid single-chip RX beamformer with non-uniform sampling for 2D-CMUT ultrasound imaging to achieve wide dynamic range of delay and small chip area," in IEEE Int. Solid-State Circuits Conf. (ISSCC) Dig. Tech. Papers, Feb. 2014, pp. 426-427.

[17] M.-C. Chen et al., "A pixel-pitch-matched ultrasound receiver for 3D photoacoustic imaging with integrated delta-sigma beamformer in 28 nm UTBB FDSOI," in IEEE Int. Solid-State Circuits Conf. (ISSCC) Dig. Tech. Papers, Feb. 2017, pp. 456-457.

[18] Y. Kim et al., "A single-chip 64-channel ultrasound RX-beamformer including analog front-end and an LUT for non-uniform ADC-sampleclock generation," IEEE Trans. Biomed. Circuits Syst., vol. 11, no. 1, pp. 87-97, Feb. 2017.

[19] M. D'Urbino et al., "An element-matched band-pass delta-sigma ADC for ultrasound imaging," in Proc. IEEE Asian Solid-State Circuits Conf. (A-SSCC), Nov. 2017, pp. 137-140.

[20] Guidance for Industry and FDA Staff Information for Manufacturers Seeking Marketing Clearance of Diagnostic Ultrasound Systems and Transducers, U.S. Food Drug Admin. (FDA), Silver Spring, MD, USA, Sep. 2008.

[21] T. Kim and S. Kim, "A $12.1 \mathrm{~mW}, 60 \mathrm{~dB}$ SNR, 8-channel beamforming embedded SAR ADC for ultrasound imaging systems," in Proc. IEEE Asian Solid-State Circuits Conf. (A-SSCC), Nov. 2017, pp. 141-144.

[22] C. Chen et al., "A $0.91 \mathrm{~mW} / \mathrm{element}$ pitch-matched front-end ASIC with integrated subarray beamforming $\mathrm{ADC}$ for miniature $3 \mathrm{D}$ ultrasound probes," in IEEE Int. Solid-State Circuits Conf. (ISSCC) Dig. Tech. Papers, Feb. 2018, pp. 186-188.

[23] Cyclone V Device Datasheet, Altera, San Jose, CA, USA, 2016.

[24] Z. Yu, M. A. P. Pertijs, and G. C. M. Meijer, "Ultrasound beamformer using pipeline-operated S/H delay stages and charge-mode summation," Electron. Lett., vol. 47, no. 18, pp. 1011-1012, Sep. 2011.

[25] D. Bera et al., "Multiline 3D beamforming using micro-beamformed datasets for pediatric transesophageal echocardiography," Phys. Med. Biol., vol. 63, no. 7, p. 075015, Apr. 2018

[26] P. Santos, G. U. Haugen, L. Løvstakken, E. Samset, and J. D'hooge, "Diverging wave volumetric imaging using subaperture beamforming," IEEE Trans. Ultrason., Ferroelectr., Freq. Control, vol. 63, no. 12, pp. 2114-2124, Dec. 2016

[27] B. D. Steinberg, "Digital beamforming in ultrasound," IEEE Trans. Ultrason., Ferroelectr, Freq. Control, vol. 39, no. 6, pp. 716-721, Nov. 1992.

[28] B. Murmann. ADC Performance Survey 1997-2017. Accessed: Jul. 16, 2018. [Online]. Available: http://web.stanford. edu/ murmann/adcsurvey.html
[29] Y. Zhu et al., "A 10-bit 100-MS/s reference-free SAR ADC in $90 \mathrm{~nm}$ CMOS," IEEE J. Solid-State Circuits, vol. 45, no. 6, pp. 1111-1121, Jun. 2010

[30] C.-C. Liu, S.-J. Chang, G.-Y. Huang, and Y.-Z. Lin, "A 10-bit 50-MS/s SAR ADC with a monotonic capacitor switching procedure," IEEE J. Solid-State Circuits, vol. 45, no. 4, pp. 731-740, Apr. 2010.

[31] M. J. Kramer, E. Janssen, K. Doris, and B. Murmann, "A 14 b 35 MS/s SAR ADC achieving $75 \mathrm{~dB}$ SNDR and $99 \mathrm{~dB}$ SFDR with loopembedded input buffer in $40 \mathrm{~nm}$ CMOS," IEEE J. Solid-State Circuits, vol. 50, no. 12, pp. 2891-2900, Dec. 2015.

[32] J. Craninckx and G. van der Plas, "A 65 fJ/conversion-step 0-to-50 MS/s 0-to- $0.7 \mathrm{~mW} 9 \mathrm{~b}$ charge-sharing SAR ADC in $90 \mathrm{~nm}$ digital CMOS," presented at the IEEE Int. Solid-State Circuits Conf. (ISSCC) Dig. Tech. Papers, 2007

[33] S.-W. M. Chen and R. W. Brodersen, "A 6-bit 600-MS/s 5.3-mW asynchronous ADC in 0.13- $\mu \mathrm{m}$ CMOS," IEEE J. Solid-State Circuits, vol. 41, no. 12 , pp. 2669-2680, Dec. 2006.

[34] A. X. Widmer and P. A. Franaszek, "A DC-balanced, partitioned-block, 8 B/10 B transmission code," IBM J. Res. Develop., vol. 27, no. 5, pp. 440-451, Sep. 1983.

[35] C. Chen, Z. Chen, Z.-Y. Chang, and M. A. P. Pertijs, "A compact $0.135-\mathrm{mW} /$ channel LNA array for piezoelectric ultrasound transducers," in Proc. Eur. Solid-State Circuits Conf. (ESSCIRC), Sep. 2015, pp. $404-407$.

[36] K. A. Ng and Y. P. Xu, "A compact, low input capacitance neural recording amplifier," IEEE Trans. Biomed. Circuits Syst., vol. 7, no. 5, pp. 610-620, Oct. 2013.

[37] B. Malki, T. Yamamoto, B. Verbruggen, P. Wambacq, and J. Craninckx, "A $70 \mathrm{~dB}$ DR 10 b 0-to- $80 \mathrm{MS} / \mathrm{s}$ current-integrating SAR ADC with adaptive dynamic range," IEEE J. Solid-State Circuits, vol. 49, no. 5, pp. 1173-1183, May 2014.

[38] J. Kuppambatti and P. R. Kinget, "Current reference pre-charging techniques for low-power zero-crossing pipeline-SAR ADCs," IEEE J. Solid-State Circuits, vol. 49, no. 3, pp. 683-694, Mar. 2014

[39] B. Razavi, Design of Analog CMOS Integrated Circuits. Boston, MA, USA: McGraw-Hill, 2001.

[40] A. G. M. Strollo, E. Napoli, and D. De Caro, "New clock-gating techniques for low-power flip-flops," in Proc. Int. Symp. Low Power Electron. Des., Rapallo, Italy, 2000, pp. 114-119.

[41] D. Schinkel, E. Mensink, E. Klumperink, E. V. Tuijl, and B. Nauta, "A double-tail latch-type voltage sense amplifier with $18 \mathrm{ps}$ setup + hold time," presented at the IEEE Int. Solid-State Circuits Conf. (ISSCC) Dig. Tech. Papers, Feb. 2007.

[42] M. Miyahara, Y. Asada, D. Paik, and A. Matsuzawa, "A low-noise selfcalibrating dynamic comparator for high-speed ADCs," in Proc. Asian Solid State Circuits Conf. (A-SSCC), Nov. 2008, pp. 269-272.

[43] H.-H. Chang, J.-W. Lin, C.-Y. Yang, and S.-I. Liu, "A wide-range delaylocked loop with a fixed latency of one clock cycle," IEEE J. Solid-State Circuits, vol. 37, no. 11, pp. 1021-1027, Aug. 2002.

[44] M. K. Chirala, P. Huynh, J. Ryu, and Y.-H. Kim, "A 128-ch $\Delta-\Sigma$ ADC based mixed signal IC for full digital beamforming wireless handheld ultrasound imaging system," in Proc. IEEE Eng. Med. Biol. Soc. (EMBC), Aug. 2015, pp. 1339-1342.

[45] G. D. Kim et al., "A single FPGA-based portable ultrasound imaging system for point-of-care applications," IEEE Trans. Ultrason., Ferroelectr., Freq. Control, vol. 59, no. 7, pp. 1386-1394, Jul. 2012.

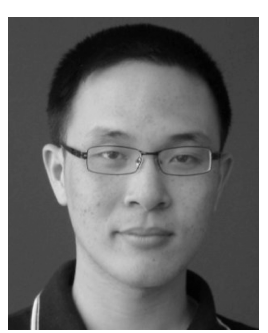

Chao Chen (S'12-M'17) received the B.Sc. degree in micro-electronics from Tsinghua University, Beijing, China, in 2010, and the M.Sc. (cum laude) and Ph.D. degrees in micro-electronics from the Delft University of Technology, Delft, The Netherlands, in 2012 and 2018, respectively.

His Ph.D. research topic was front-end applicationspecified integrated circuit (ASIC) design for 3-D medical ultrasound imaging. Since 2017, he has been a Senior Analog IC Designer with Butterfly Network Inc., Guilford, CT, USA, where he focuses on the development of next-generation ASICs for advanced medical ultrasound systems. His current research interests include integrated circuits for medical ultrasound imaging, low-power sensor interfaces, and high-resolution data converters.

Dr. Chen was a recipient of the Huygens Scholarship in 2011, the ISSCC 2013 STGA Award, and the winner of the IEEE IUS 2017 Student Paper Competition Award. He was also a co-recipient of the A-SSCC 2017 Best Student Paper Award. 


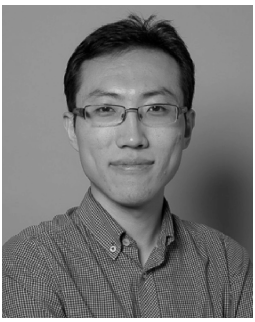

Zhao Chen (S'15) received the B.Sc. degree in micro-electronics from Fudan University, Shanghai, China, in 2011, and the M.Sc. degree in electrical and electronic engineering from Imperial College, London, U.K., in 2012. He is currently pursuing the $\mathrm{Ph} . \mathrm{D}$. degree in electrical engineering with the Electronic Instrumentation Laboratory, Delft University of Technology, Delft, The Netherlands, with a focus on ASIC design for 3-D medical ultrasound imaging.

His current research interests include analog- and mixed-signal electronics, especially for biomedical applications.

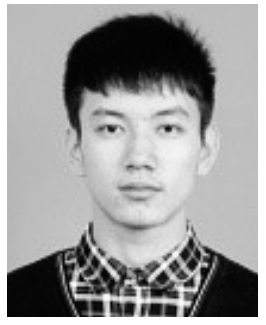

Mingliang Tan received the B.S. degree in electronic science and technology from Northeastern University, Shenyang, China, in 2014, and the M.S. degree in micro-electronics from the Delft University of Technology, Delft, The Netherlands, in 2016, where he is currently pursuing the Ph.D. degree in electrical engineering with the Electronic Instrumentation Laboratory, with a focus on ASIC design for medical ultrasound imaging.

His current research interests include analog and mixed-signal electronics, especially for biomedical applications.

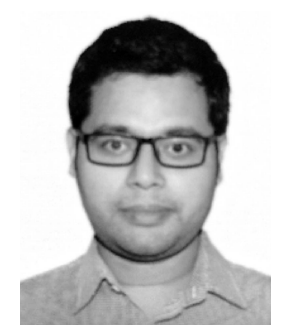

Deep Bera received the B.Tech. degree in computer science and engineering from the West Bengal University of Technology, Kolkata, India, in 2006, the M.S. degree in electronics and electrical engineering from IIT Kharagpur, Kharagpur, India, in 2011, and the Ph.D. degree in biomedical engineering from the Department of Biomedical Engineering, Erasmus Medical Center, Rotterdam, The Netherlands, in 2018.

From 2010 to 2012, he was a Scientist with the Samsung Advanced Institute of Technology, Suwon, South Korea, where he worked on machine learning and real-time biomedical signal processing on physiological signals. From 2012 to 2013, he was a Scientist with Philips Research, where he was involved in research related to clinical decision support systems and risk prediction modeling for Cardiovascular disease in Chinese population. He is currently a Senior Scientist with Philips Research, Bengaluru, India, working on ultrasound beamforming and elastography. His current research interests include 2-D/3-D beamforming for medical ultrasound, especially for echocardiography.

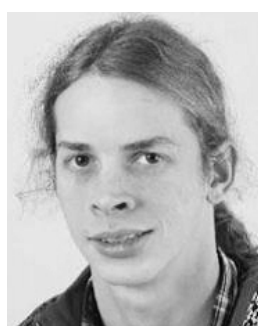

Emile Noothout received the bachelor's degree from the Intermediate Technical School for Mechanics, Dordrecht, The Netherlands, in 2004.

From 2004 to 2006, he was with Leidse Instrumentmaker School, Leiden, The Netherlands, where he studied for Research Instrument Maker. From 2007 to 2013, he was a Research Instrument Maker with TNO, Delft, The Netherlands, where he developed hardware for space and lithography. Since 2008 , he has been involved in developing and building ultrasound transducers for the petrochemical industries. Since 2013, he has been with the Delft University of Technology, Delft, where he is involved in the development of medical ultrasound transducers and research assistance.

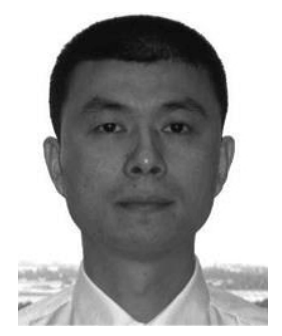

Zu-Yao Chang received the M.Sc. degree in electrical engineering from the Delft University of Technology, Delft, The Netherlands, in 2003.

Since 2003, he has been a Staff Member with the Electronic Instrumentation Laboratory, Delft University of Technology, where he works on impedance measurement systems and smart sensor systems.

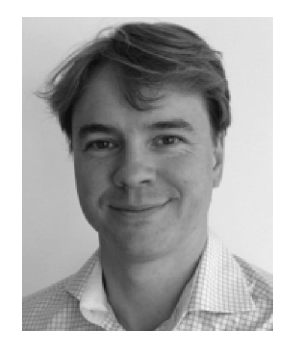

Hendrik J. Vos (M'14) received the M.Sc. degree in applied physics from the Delft University of Technology, Delft, The Netherlands, in 2004, and the Ph.D. degree from the Department of Biomedical Engineering, Erasmus Medical Center, Rotterdam, The Netherlands, in 2010.

He was a Postmaster Researcher with the University of Florence, Florence, Italy, and a Contract Researcher with the Petrochemical Industry, where he worked on cutting-edge ultrasonic solutions. He is currently an Assistant Professor with Erasmus MC and received a Dutch NWO-TTW-VIDI personal grant in 2018. His current research interests include acoustical array technology for biomedical imaging in all its aspects: transducers, 2-D and 3-D beamforming, cardiac shear waves, ultrafast Doppler, contrast imaging, and related subclinical and clinical studies.

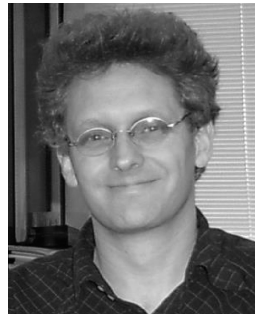

Johan G. Bosch (M'07) received the M.Sc. degree in electrical engineering from the Eindhoven University of Technology, Eindhoven, The Netherlands, in 1985, and the Ph.D. degree in medicine from the Leiden University Medical Center, Leiden, The Netherlands, in 2006.

$\mathrm{He}$ is currently an Associate Professor and a Staff Member with the Department of Biomedical Engineering, Thoraxcenter, Erasmus Medical Center, Rotterdam, The Netherlands. His current research interests include 2-D and 3-D echocardiographic image processing/analysis and transducer development.

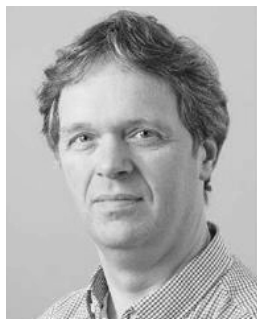

Martin D. Verweij (M'10) received the M.Sc. (cum laude) and $\mathrm{Ph} . \mathrm{D}$. degrees in electrical engineering from the Delft University of Technology, Delft, The Netherlands, in 1988 and 1992, respectively.

From 1993 to 1997, he was a Research Fellow with the Royal Netherlands Academy of Arts and Sciences, Amsterdam, The Netherlands. In 1998, he became an Associate Professor with the Laboratory of Electromagnetic Research, Delft University of Technology, and joined the Laboratory of Acoustical Wavefield Imaging in 2011. His current research interests include dedicated transducer design, beamforming algorithms, and the theoretical modeling and numerical simulation of medical ultrasound.

Dr. Verweij is an Associate Editor of the Journal of the Acoustical Society of America. 


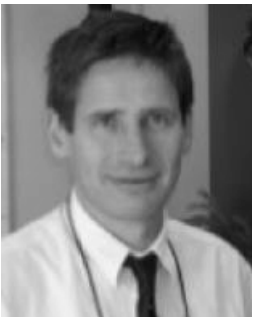

Nico de Jong (A'97-M’09) received the M.Sc. degree in physics from the Delft University of Technology, Delft, The Netherlands, in 1978, and the $\mathrm{Ph} . \mathrm{D}$. degree in acoustic properties of ultrasound contrast agents from the Erasmus Medical Center, Rotterdam, The Netherlands, in 1993.

Since 1980, he has been a Staff Member with Thoraxcenter, Erasmus Medical Center. In 2003, he joined the University of Twente, Enschede, The Netherlands, as a part-time Professor. Over the last five years, he has given more than 30 invited lectures and has given numerous scientific presentations for international industries. He teaches in technical universities and at the Erasmus Medical Center $\mathrm{He}$ has been the promotor of $21 \mathrm{Ph} . \mathrm{D}$. students and is currently supervising 11 Ph.D. students. Since 2011, he has been a Professor of molecular ultrasonic imaging and therapy with the Erasmus Medical Center and also with the Delft University of Technology.

Dr. de Jong is an Organizer of the Annual European Symposium on Ultrasound Contrast Imaging held at Rotterdam and attended by approximately 175 scientists from universities and industries all over the world. $\mathrm{He}$ is on the Safety Committee of the World Federation of Ultrasound in Medicine and Biology and is an Associate Editor of Ultrasound in Medicine and Biology, and has been a Guest Editor of special issues of several journals.

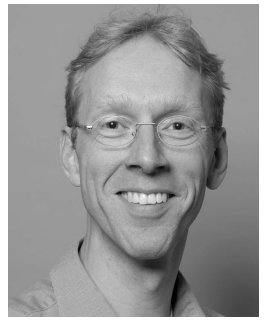

Michiel A. P. Pertijs (S'99-M'06-SM'10) received the M.Sc. and Ph.D. degrees (cum laude) in electrical engineering from the Delft University of Technology, Delft, The Netherlands, in 2000 and 2005, respectively.

From 2005 to 2008, he was with National Semiconductor, Delft, where he designed precision operational amplifiers and instrumentation amplifiers. From 2008 to 2009, he was a Senior Researcher with the IMEC/Holst Centre, Eindhoven, The Netherlands. In 2009, he joined the Electronic Instrumentation Laboratory, Delft University of Technology, where he is currently an Associate Professor. He heads a research group focusing on integrated circuits for medical ultrasound and energy-efficient smart sensors. He has authored or co-authored two books, three book chapters, 12 patents, and over 90 technical papers.

Dr. Pertijs is a member of the Technical Program Committee of the European Solid-State Circuits Conference (ESSCIRC). He was a recipient of the International Solid-State Circuits Conference (ISSCC) 2005 Jack Kilby Award for Outstanding Student Paper and the Journal OF Solid-STATE Circuits (JSSC) 2005 Best Paper Award. For his Ph.D. research on highaccuracy CMOS smart temperature sensors, he received the 2006 Simon Stevin Gezel Award from the Dutch Technology Foundation STW. In 2014, he was elected Best Teacher of the EE Program at the Delft University of Technology. He served as an Associate Editor for the IEEE JSSC, and also served on the program committees of ISSCC and the IEEE Sensors Conference. 